\title{
The Impact of Electroacupuncture Early Intervention on the Brain Lipidome in a Mouse Model of Post- traumatic Stress Disorder
}

\section{Cuihong Zhou}

Xijing Hospital

Fen Xue

Xijing Hospital

Qingqing Shi

Xijing Hospital

Shanshan Xue

Xijing Hospital

Tian Zhang

Xijing Hospital

Xinxu Ma

Xijing Hospital

Lisheng Yu

Xi'an Medical University

Chuang Liu

Xijing Hospital

Huaning Wang

Xijing Hospital

Zhengwu Peng ( $\sim$ pengzhengwu1446@163.com)

Xijing Hospital https://orcid.org/0000-0002-8185-0538

\section{Research Article}

Keywords: Electroacupuncture, posttraumatic stress disorder, lipidomics, hippocampus, prefrontal cortex, mouse model

Posted Date: November 8th, 2021

DOI: https://doi.org/10.21203/rs.3.rs-1038190/v1

License: (a) (i) This work is licensed under a Creative Commons Attribution 4.0 International License.

Read Full License 
Version of Record: A version of this preprint was published at Frontiers in Molecular Neuroscience on February 10th, 2022. See the published version at https://doi.org/10.3389/fnmol.2022.812479. 


\section{Abstract}

The neuroprotective effect of electroacupuncture (EA) treatment has been well studied; growing evidence suggests that changes in lipid composition may be involved in the pathogenesis of posttraumatic stress disorder (PTSD) and may be a target for treatment. However, the influence of early EA intervention on brain lipid composition in patients with PTSD has never been investigated. Using a modified single prolonged stress (mSPS) model in mice, we assessed the anti-PTSD-like effects of early intervention using EA and evaluated changes in lipid composition in the hippocampus and prefrontal cortex (PFC) using a mass spectrometry-based lipidomic approach. mSPS induced changes in lipid composition in the hippocampus, notably in the content of sphingolipids, glycerolipids, and fatty acyls. These lipid changes were more robust than those observed in the PFC. Early intervention with EA after mSPS ameliorated PTSD-like behaviors and partly normalized mSPS-induced lipid changes, notably in the hippocampus. Cumulatively, our data suggest that EA may reverse mSPS-induced PTSD-like behaviors due to regionspecific regulation of the brain lipidome, providing new insights into the therapeutic mechanism of EA.

\section{Introduction}

Posttraumatic stress disorder (PTSD) is a stressor-related disorder characterized by avoidance, reexperiencing of trauma, and hyperarousal symptoms, and affects approximately $7-10 \%$ of the general population [1-3]. It is worth noting that the prevalence of PTSD increased significantly after the start of the COVID-19 pandemic [4, 5]. PTSD is often associated with other psychiatric symptoms and functional impairment. The disorder may persist over the patient's lifetime and entail costs higher than those associated with other anxiety disorders [6-8]. Currently, the main treatment strategy for PTSD is psychological intervention combined with pharmacotherapy. However, psychological intervention provides only partial benefits [9]. Moreover, the benefits of drug treatments decline over time and drug use carries a risk of withdrawal syndromes and relapse [10]. Investigation of the pathogenesis of PTSD and the development of new approaches for treating it are thus urgently needed [11, 12].

Electroacupuncture $(E A)$ is a modified form of traditional acupuncture. The latter is a technique of traditional Chinese medicine typically used in the field of complementary and alternative medicine [13]. Previous studies have mainly focused on the analgesic effect of EA [14]. However, multiple studies have found that electroacupuncture not only has neuromodulatory effects $[15,16]$, but can also ameliorate the symptoms of several neuropsychiatric diseases [17-19]. Interestingly, a large number of studies have reported that EA exerts neuroprotective effects by regulating the structure and function of the hippocampus and prefrontal cortex (PFC) [20-22], which are regions of central importance in PTSD due to their prominent role in the neuroendocrine stress response and memory [23-25]. Clinical and preclinical studies have previously reported that EA is protective against PTSD [26, 27], and our previous work further found that early intervention or pretreatment using EA could prevent PTSD-like behaviors in a rat model of PTSD $[28,29]$. Taken together, these results show that EA treatment is potentially therapeutic in cases of PTSD. 
Lipids play an important role in maintaining and regulating brain development and function. Such functions include learning/memory and emotional behavior [30-32]. The involvement of lipids in modulating synaptic physiology, receptor pharmacology, energy generation, and brain metabolism has largely been established [33-35]. Recently, lipidomic alterations have been reported in psychiatric disorders, including bipolar disorder [36, 37], schizophrenia [38], and anxiety disorder [39]. Peripheral lipids may also be promising biomarker candidates to assist in the differential diagnosis of mild traumatic brain injury and PTSD [40]. However, to our knowledge, the changes in the brain lipidome occurring in PTSD and the link between the neuroprotective effects of EA and lipidomic alterations remain elusive.

Considering the above, we aimed to determine the influence of early EA intervention on the lipidomic composition of the hippocampus and PFC in a mouse model of modified, single prolonged stress (mSPS), which can produce robust symptoms and enhanced conditioned and sensitized fear responses that mimic PTSD [41]. We also aimed to determine the lipid changes characteristic of PTSD and EA treatment.

\section{Materials And Methods}

\section{Animals}

Adult male C57BL/6 mice (8 weeks old, weighing 18-22 g) were obtained from the Fourth Military Medical University Animal Center (Xi'an, China). The mice were group-housed at 4 per cage in wirebottomed cages at $20-25^{\circ} \mathrm{C}$ and maintained on a $12 \mathrm{~h}$ light/12 $\mathrm{h}$ dark daily cycle (lights on from 8:00 AM to 8:00 PM). Food and water were available ad libitum and all experiments were conducted during the light phase. The experimental procedures of this study were in accordance with the National Institutes of Health Guide for the Care and Use of Laboratory Animals and were approved by the Animal Use and Protection Committee of the Fourth Military Medical University.

\section{Experimental design}

As shown in Figure 1A, after seven days of acclimatization, 32 mice were randomly assigned to the following four groups ( $n=8 /$ group): Sham, EA, PTSD, and PTSD+EA. Mice in the PTSD and PTSD+EA groups were treated with mSPS. Subsequently, mice in the EA and PTSD+EA groups were stimulated by EA at a frequency of $2 / 15 \mathrm{~Hz}$ and an intensity of $1 \mathrm{~mA}$ for $30 \mathrm{~min}$ each day, for 7 consecutive days. Mice in the Sham and PTSD groups were administered false stimulation (EA treatment without application of electric current) for 30 min each day for 7 consecutive days, and then handled in the home cage for one week. The researchers performing the behavioral testing were blinded to the animals' group allocations, and the behavioral tests were administered one week after the final intervention. Subsequently, the mice were sacrificed and the PFC and hippocampus were immediately collected in liquid nitrogen for later lipidomic analyses by high performance liquid chromatography-tandem mass spectrometry (HPLC$\mathrm{MS} / \mathrm{MS})$. 
The modified SPS paradigm was as described in a previous study [42], with some modifications. Briefly, mice were restrained for $2 \mathrm{~h}$ and then immediately administered forced swimming for 20 min in an acrylic cylindrical tank $\left(10 \mathrm{~cm}\right.$ diameter, $25 \mathrm{~cm}$ height, water temperature $20-24^{\circ} \mathrm{C}$ ) filled approximately twothirds with water. Following gentle drying, the mice were subsequently exposed to diethyl ether until they lost consciousness for 2-3 min. After a 15-min recovery, the mice were exposed to a single electric foot shock ( $0.75 \mathrm{~mA}$ for $2 \mathrm{~s})$ in a square box ( $360 \mathrm{~mm}$ width $\times 360 \mathrm{~mm}$ height $\times 360 \mathrm{~mm}$ depth) with a floor of stainless-steel rods and walls of aluminum and acrylic. Subsequently, the mice were returned to their home cages.

\section{EA treatment}

The electroacupuncture treatment method was as described previously [43]. Mice were anesthetized with isoflurane (1.5 × minimum alveolar concentration) during EA stimulation and the "Bai hui" acupoint (GV20), which is located at the intersection of the sagittal midline and the line linking the ears, was stimulated for 30 min daily at a frequency of $2 / 15 \mathrm{~Hz}$ and an intensity of $1 \mathrm{~mA}$ (waveform: dilatational wave) with a G6805-2 electric acupuncture apparatus (No. 227033; Qingdao Xinsheng Ltd.). False stimulation was performed at the same acupoint without electricity (Mice receiving false stimulation were also anesthetized with isoflurane).

\section{Behavioral testing}

Behavioral testing occurred 14 days after mSPS (see Figure 1A). Animals were acclimated to a separate experimental room for at least 30 min prior to each test. The open field test was conducted prior to the elevated plus maze test on the same day, while the fear conditioning test was performed $24 \mathrm{~h}$ after the elevated plus maze test. All experiments were conducted under low light conditions to minimize anxiety effects and the test area was cleaned with $75 \%$ ethanol between tests.

Open-field test (OFT)

Based on a previous study [44], the OFT was performed in an unfamiliar soundproof box $(50 \times 50 \times 50$ $\mathrm{cm})$ made of white polycarbonate. The floor had black grid marks dividing the behavioral arena into 36 squares comprising 16 central and 20 peripheral squares. Animals were placed in the center of the openfield box and activity was recorded for a period of $5 \mathrm{~min}$ by a video camera positioned directly above the arena. The recordings were analyzed by the open-field activity software Top Scan (Clever Sys Inc., USA). The time spent and distance travelled in the central squares and entire arena were measured.

Elevated-plus maze test (EPMT)

The EPMT was used to detecting anxiety-like behavior in mice [45]. The maze apparatus (Dig Behav, Ji liang Co. Ltd., Shanghai, China) consisted of two opposing open arms of $35 \times 6 \mathrm{~cm}$ and two opposing enclosed arms of $35 \times 6 \mathrm{~cm}$, all elevated $50 \mathrm{~cm}$ above the floor. During a test, a mouse was placed in the center square of the maze facing an open arm and its behavior recorded for $5 \mathrm{~min}$ by an automatic 
analyzing system (Top Scan, Clever Sys Inc., USA). The number of arm entries and the time spent in open arms were used as indices of anxiety.

Fear conditioning response (FCR) test

Conditioned fear behavior was examined by the FCR test as described in previous reports [42]. The experiments were performed in two dissimilar chambers: a shock chamber (Context A: a rectangular box with floors made of stainless-steel rods and walls of aluminum and acrylic) and a neutral-context chamber (Context B: a rectangular box with a white acrylic floor and an acrylic frame roof). The contextual fear conditioning paradigm and the cued fear conditioning paradigm each consisted of one training and two test sessions. For training, mice were acclimated to the shock chamber (Context A) for $180 \mathrm{~s}$, then presented with a pure tone $(28 \mathrm{~s}, 1 \mathrm{kHz}, 80 \mathrm{~dB})$ immediately followed by a foot shock ( $2 \mathrm{~s}$, $0.75 \mathrm{~mA}$ ). The tone-foot-shock pairing was delivered twice followed by a 90 -s rest period, also in the chamber. Twenty-four hours later, mice were placed in the contextual chamber (Context A) for 5 min, without exposure to tone or foot shock, for the contextual fear response test. The cued fear response test was performed $1 \mathrm{~h}$ later. In this test, mice were acclimated to the neutral chamber (Context B) for $3 \mathrm{~min}$. Subsequently, a neutral tone $(4 \mathrm{kHz}, 80 \mathrm{~dB})$ was presented without foot-shock for 3 min. Mice were returned to the home cage after resting in the neutral chamber (Context B) for another $60 \mathrm{~s}$. Freezing behavior was recorded and analyzed using a computerized automatic analysis system (Freezing Scan, Clever Sys Inc., Reston, VA, USA).

\section{Tissue collection}

Mice were euthanized by cervical dislocation within $4 \mathrm{~h}$ of the end of behavioral testing. The brains were subsequently removed and the PFC and hippocampi were dissected on ice, then precisely weighed, frozen in liquid nitrogen, and stored until use.

\section{Sample extraction}

Lipids were extracted with methyl tert-butyl ether (MTBE) as described in a previous study [46]. Briefly, 30 mg samples were spiked with appropriate amounts of internal lipid standards $(20 \mu \mathrm{L})$ and then homogenized with $200 \mu \mathrm{L}$ of water and $240 \mu \mathrm{L}$ of methanol. Subsequently, $800 \mu \mathrm{L}$ of MTBE were added, and samples were ultrasonicated for $20 \mathrm{~min}$ at $4^{\circ} \mathrm{C}$, then incubated at $25^{\circ} \mathrm{C}$ for $30 \mathrm{~min}$. To separate the organic components, the solution was centrifuged at $10^{\circ} \mathrm{C}$ for $15 \mathrm{~min}$ at $14,000 \times \mathrm{g}$. The upper layer, which was the organic solvent phase, was collected and the solvent evaporated under nitrogen. The solutes were stored at $-80^{\circ} \mathrm{C}$ until use.

\section{Lipid analysis by HPLC-MS/MS}

Reverse-phase chromatography was selected for ultra-HPLC separation using a CSH C18 column (1.7 $\mu \mathrm{m}$, $2.1 \times 100 \mathrm{~mm}$, Waters). The lipid extracts were re-dissolved in $200 \mu \mathrm{L}$ of $90 \%$ isopropanol/acetonitrile and centrifuged at $14,000 \times g$ for 15 min; finally, $3 \mu \mathrm{L}$ of each sample was injected for analysis. Solvent A was acetonitrile-water $(6: 4, \mathrm{v} / \mathrm{v})$ containing $0.1 \%$ formic acid and $0.1 \mathrm{mM}$ ammonium formate; solvent $B$ was 
acetonitrile-isopropanol $(1: 9, \mathrm{v} / \mathrm{v})$ also containing $0.1 \%$ formic acid and $0.1 \mathrm{mM}$ ammonium formate. The initial mobile phase was $30 \%$ solvent $B$ at a flow rate of $300 \mu \mathrm{L} / \mathrm{min}$, which was held for two min, then linearly increased to $100 \%$ solvent B over 23 min, followed by equilibration in $5 \%$ solvent B for 10 min. The autosampler was maintained at $10^{\circ} \mathrm{C}$. To avoid bias due to instrumentation errors, multiple samples were analyzed concurrently and signal fluctuations were detected in random order, even during sample analysis. Sampling of the queue was performed every eight samples using one of the quality control (QC) samples to monitor the stability of the analysis and evaluate the reliability of the experimental data.

After separation by ultra-HPLC, samples were analyzed using a Q Exactive $\mathrm{T}^{\mathrm{TM}}$ plus mass spectrometer (Thermo Fisher Scientific, Waltham, USA) using the following parameters. For positive-ion mode: heater temperature, $300^{\circ} \mathrm{C}$; sheath gas flow rate, 45 arbitrary units (arb); auxiliary gas flow rate, 15 arb; sweep gas flow rate, $1 \mathrm{arb}$; spray voltage, $3.0 \mathrm{kV}$; capillary temperature, $350^{\circ} \mathrm{C}$; S-Lens radio frequency (RF) level, $50 \%$; and MS1 scan range, $200-1800 \mathrm{~m} / \mathrm{z}$. For negative-ion mode: heater temperature, $300^{\circ} \mathrm{C}$; sheath gas flow rate, 45 arb; auxiliary gas flow rate, 15 arb; sweep gas flow rate, 1 arb; spray voltage, $2.5 \mathrm{kV}$; capillary temperature, $350^{\circ} \mathrm{C}$; S-Lens RF level, $60 \%$; and MS2 scan range, $250-1800 \mathrm{~m} / \mathrm{z}$.

\section{Lipid identification using LipidSearch ${ }^{\mathrm{TM}}$}

LipidSearch (Thermo Fisher Scientific, Walthamcity, country, USA) is a search engine used to identify lipid species based on MS/MS data. LipidSearch contains data on $>30$ lipid classes and >1,500,000 ion fragments. The mass tolerances for both molecular precursors and fragment ions were set to $5 \mathrm{ppm}$. The displayed product ion threshold was set at five, and grades A, B, C, D were all used in the identification (ID) quality filtering. All lipid classes in the database, including 71 sub-species, were chosen for identification. Adducts of $\mathrm{H}^{+}$and $\mathrm{NH}_{4}{ }^{+}$were selected for positive-mode searches, and adducts of $\mathrm{H}^{-}$and $\mathrm{CH}_{3} \mathrm{COO}^{-}$were selected for negative-mode searches, since ammonium acetate was used in the mobile phases.

\section{Statistical analysis}

Statistical analyses were performed using SPSS v.19.0 software (SPSS Inc., Chicago, IL, USA). The results of behavioral testing and the characterizations of lipid compositions are presented as mean \pm standard deviation (SD), and were evaluated by one- or two-way analysis of variance (ANOVA) followed by a Bonferroni post-hoc test for pairwise comparisons. A $P$-value $<0.05$ was deemed statistically significant. The correlations between lipid species levels and behaviors were analyzed using the Pearson correlation.

The raw lipidomic data were processed using LipidSearch software for peak recognition, lipid-peak extraction (secondary appraisal), peak alignment, and quantitative processing. After normalization and integration using the Perato scaling method, the processed data were imported into SIMPCA-P 14.1 (Umetrics, Umea, Sweden) for multivariate statistical analysis, which included principal component 
analysis, partial least squares discriminant analysis, and orthogonal partial least squares discriminant analysis. Lipids with significant differences were identified based on a combination of 1) statistically significant thresholds of variable influence on projection values (obtained from orthogonal partial least squares discriminant analysis and two-tailed Student's $t$-tests) and 2) mapping of volcano, hierarchicalcluster, and correlation analyses using R software [47].

\section{Results}

\section{EA treatment ameliorates anxiety-like behaviors and fear learning defects in mSPS-treated mice}

First, we determined the effect of EA treatment on PTSD-like behaviors (Figure 1A). Two-way ANOVA revealed that mSPS and EA treatment did not induce any motor impairment in mice because in the OFT, we observed no significant differences in the total distance traveled in either the mSPS or the EA treatment factors ( $F=2.320, P=0.139$; and $F=0.270, P=0.871$; respectively; Figure 1B, $C)$. However, we observed significant differences in the time spent in the center squares in the OFT for the mSPS factor ( $F$ $=4.348, P=0.046$, Figure 1D), as well as in the open arms of the EPMT (Figures 1E, F) for both the mSPS $(F=10.401, P<0.01)$ and EA factors $(F=7.373, P=0.011)$. In addition, for the mSPS factor, all differences in freezing time were significant for both contextual fear $(F=10.587, P<0.01$, Figure $1 \mathrm{G})$ and cued fear $(F=18.389, P<0.01$, Figure $1 \mathrm{H})$. Post hoc comparisons further showed that mSPS markedly reduced the time spent in the center in the OFT and the time spent in the open arms in the EPMT (PTSD $v s$. Sham, $P<0.05$ ), and that these measures were ameliorated after EA treatment (PTSD+EA vs. PTSD, $P$ $<0.05)$. Additionally, the PTSD group showed a significant increase in freezing time in both contextual fear and cued fear conditioning tests when compared with the Sham group $(P<0.01)$. Moreover, EA treatment significantly decreased freezing time and enhanced fear learning in the PTSD-treated mice (PTSD+EA vs. PTSD, $P<0.05$, Figure 1G, H). These results suggest that EA treatment can ameliorate anxiety-like behaviors and fear learning defects in PTSD model mice.

\section{EA treatment effect: hippocampal lipid classes}

As shown in Figure 2, we found no statistical intergroup differences in total lipid levels $(F=1.598, P=$ 0.2122 ) in the hippocampus (Figure 2A). However, in this structure, we observed significant differences for the mSPS factor in the concentrations of the following: diglyceride (DG; $F=44.152, P<0.001$ ), triglyceride (TG; $F=6.226, P=0.019$ ), sphingomyelin (SM; $F=20.162, P<0.001$ ), ceramides (Cer; $F=$ 5.807, $P=0.023$ ), gangliosides (GM1; $F=9.702, P=0.004)$, cardiolipin ( $C L ; F=28.410, P<0.001)$, lysophosphatidylglycerol (LPG; $F=13.624, P=0.001)$, lysophosphatidylinositol (LPI; $F=11.034, P<$ 0.01), lysophosphatidylserine (LPS; $F=14.791, P=0.001$ ), phosphatidic acid (PA; $F=12.734, P=0.001$ ), phosphatidylethanolamine (PE; $F=37.813, P<0.001$ ), phosphatidylglycerol (PG; $F=37.426, P<0.001$ ), phosphatidylinositol (Pl; $F=16.968, P<0.001$ ), acyl carnitines (AcCa; $F=23.807, P<0.001$ ), sulfoquinovosyldiacylglycerol (SQDG; $F=21.967, P<0.001$ ), monogalactosyldiacylglycerol (MGDG; $F=$ 47.718, $P<0.001$ ), and coenzyme (Co; $F=45.690, P<0.001)$. In addition, we observed significant differences for the EA treatment factor in the concentrations of the following: TG $(F=60.011, P<0.001)$, GM1 $(F=12.357, P=0.002), \operatorname{SM}(F=43.338, P<0.001), \operatorname{Cer}(F=14.462, P<0.01)$, sphingosine (So; $F=$ 
15.388, $P=0.001), \mathrm{CL}(F=44.882, P<0.001)$, lysophosphatidylethanolamine (LPE; $F=5.653, P=0.024)$, LPG $(F=13.004, P=0.001)$, LPS $(F=30.514, P<0.001)$, PA $(F=4.757, P=0.038), \operatorname{PE}(F=19.787, P<$ 0.001), $\mathrm{PG}(F=19.259, P<0.001)$, AcCa $(F=18.013, P<0.001)$, fatty acid (FA; $F=34.261, P<0.001)$, digalactosyldiacylglycerol (DGDG; $F=27.467, P<0.001)$, SQDG $(F=43.754, P<0.001)$, MGDG $(F=$ 95.459, $P<0.001)$, and Co $(F=22.278, P<0.001)$. Post hoc comparisons revealed that mice in the PTSD group exhibited significantly elevated levels of DG, TG, Cer, GM1, LPS, lysophosphatidylcholine (LPC), PI, and PE but reduced levels of SM, CL, LPI, FA, AcCa, and Co (PTSD vs. Sham, $P<0.05$ ). EA treatment effectively deceased the levels of Cer, GM1, LPS, PE, and TG and increased the levels of SM, CL, AcCa, FA, and Co (PTSD+EA vs. PTSD, $P<0.05$ ). However, the changes in DG, LPI, LPC, and PI induced by mSPS in the hippocampus were not normalized by EA treatment.

Correlation analysis showed that the time spent in the center squares in the OFT was positively correlated with levels of AcCa, CL, LPI, SM, Co, and DGDG but negatively correlated with levels of TG, LPS, and PE. The time spent in the open arms in the EPMT was positively correlated with levels of FA, CL, LPG, LPI, PC, SM, Co, and DGDG but negatively correlated with levels of DG, TG, LPC, LPS, PE, Cer, and GM1. Moreover, the contextual freezing time recorded in the FCR test was positively correlated with levels of DG, LPC, LPS, PE, and PI but negatively correlated with levels of AcCa, FA, CL, LPG, LPI, SM, and Co. The cued FCR freezing time was positively correlated with levels of DG, LPC, LPS, PE, PI, and Cer but negatively correlated with levels of AcCa, FA, CL, LPI, and Co (Figure 4A and Table S1). These results suggest that hippocampal levels of AcCa, FA, CL, LPI, SM, and Co are negatively correlated with the severity of PTSD, while levels of DG, LPC, PE, LPS, and Cer are positively correlated with severity.

\section{EA treatment effect: PFC lipid classes}

As shown in Figure 3, we observed significant intergroup differences in total lipid levels in the PFC $\left(F_{3,28}=\right.$ 39.91, $P<0.001$, Figure 3A); mSPS treatment decreased the total lipid concentration in this region (PTSD vs. Sham, $P<0.01)$. Moreover, we found significant differences for the mSPS factor in the levels of the following lipids in the PFC: FA $(F=23.768, P<0.001)$, wax esters (WE; $F=16.173, P<0.001)$, Cer $(F=$ 16.882, $P<0.001$ ), simple Glc series (CerG2GNAc1; $F=6.514, P=0.016)$, GM1 $(F=12.544, P=0.001)$, sphingomyelin (phSM; $F=15.960, P<0.001)$, So $(F=6.030, P=0.021), \mathrm{CL}(F=16.853, P<0.001), \operatorname{LPI}(F$ $=20.337, P<0.001)$, LPS $(F=14.209, P=0.001), \mathrm{PC}(F=31.914, P<0.001), \mathrm{PE}(F=12.559, P=0.001)$, $\mathrm{PG}(F=59.824, P<0.001), \mathrm{PI}(F=23.150, P<0.001)$, phosphatidylserine $(\mathrm{PS} ; F=134.871, P<0.001)$, $\operatorname{LPE}(F=15.163, P<0.01), \mathrm{DG}(F=44.152, P<0.001)$, SQDG $(F=10.472, P=0.003)$, and MGDG $(F=$ 12.354, $P=0.002)$. We also found significant differences for the EA treatment factor in the concentrations of: FA $(F=17.552, P<0.001)$, WE $(F=27.347, P<0.001)$, $\operatorname{Cer}(F=4.422, P=0.045)$, glucosylceramides (CerG1; $F=9.916, P=0.004), \operatorname{GM} 1(F=14.835, P=0.001)$, phSM $(F=27.825, P<$ $0.001)$, SM $(F=14.343, P=0.001)$, So $(F=11.571, P=0.002)$, LPI $(F=47.752, P<0.001)$, LPS $(F=$ $10.774, P=0.003), \mathrm{PA}(F=16.878, P<0.001), \mathrm{PC}(F=25.379, P<0.001), \mathrm{PE}(F=17.896, P<0.001), \mathrm{PG}$ $(F=38.679, P<0.001)$, PS $(F=16.869, P<0.001)$, monogalactosyl monoacylglycerol (MGMG; $F=$ 33.097, $P<0.001)$, and MGDG $(F=11.254, P=0.002)$. Post hoc comparisons further revealed that mice in the PTSD group exhibited significantly decreased levels of AcCa, PS, LPG, CL, LPS, PA, PG, and Co and 
increased levels of LPE, LPC, and DG (PTSD vs. Sham, $P<0.05)$. EA treatment increased the levels of AcCa and LPG (PTSD+EA vs. PTSD, $P<0.05$ ). Although the changes in PS, CL, LPS, PA, PG, Co, LPE, and DG induced by mSPS in the PFC were not normalized by EA treatment, other changes were, and we found no significant differences in the levels of LPE, CL, PG, and Co between PTSD+EA and Sham.

Correlation analysis showed that the time spent in the center squares in the OFT was positively correlated with levels of AcCa, FA, CL, and PS in the PFC, but negatively correlated with the level of LPE. The time spent in the open arms of the EPMT was positively correlated with levels of FA, CL, PG, phSM, and Co but negatively correlated with the level of DG. The contextual freezing time in FCR was positively correlated with the level of DG but negatively correlated with the levels of AcCa, LPG, LPS, PG, PS, Cer, phSM, and SQDG. The cued freezing time in the FCR was positively correlated with DG and LPE but negatively correlated with levels of LPG, LPS, PG, PS, and Cer (Figure 4B and Table S2). These results suggest that the levels of PS and PG in the PFC negatively correlate with the severity of PTSD, and the level of DG positively correlates with severity.

\section{EA treatment effect: fatty acid composition}

As shown in Figure 5, mSPS treatment led to a significant alteration in the fatty acyl chain profile of lipids in the hippocampus and PFC. In the hippocampus, in the PTSD group, levels of long-chain fatty acyls with 38 carbons (38C, Figure 5A) and levels of polyunsaturated fatty acyls with 4 double bonds and $>6$ double bonds were increased, while levels of polyunsaturated fatty acyls with 3 double bonds were decreased (Figure 5B). Notably, these changes were ameliorated by EA treatment. On the other hand, in the PFC, levels of long-chain fatty acyls with $>32$ carbons $(38 \mathrm{C}$ ) and levels of total unsaturated fatty acids were decreased in the PTSD group, and these changes were not attenuated after EA treatment (Figure 5C, D).

\section{Characteristic lipid species in the brain and their correlation with PTSD-like behaviors}

Lipidomic profiling at the species level revealed additional intergroup changes in the concentrations of lipids in the hippocampus and PFC. As shown in Figure 6A-C and Table S3, concentrations of 60 lipids such as $\mathrm{PC}(33: 0)+\mathrm{H}, \mathrm{DG}(34: 1 \mathrm{e}),+\mathrm{Na}$ and $\mathrm{CL}(18: 2 / 20: 4 / 16: 0 / 20: 4)-\mathrm{H}$ were decreased and those of 181 lipids such as TG(16:0/18:1/20:1)+NH4, $\mathrm{PS}(42: 7 \mathrm{p})-\mathrm{H}$, and TG(17:0/18:1/18:1)+NH4 were increased in the hippocampus in the PTSD group compared with the Sham group (Figure 6A). Concentrations of 24 lipids such as DG(34:1e)+Na, LPI(18:0)-H, and TG(18:1/18:1/18:3)+NH4 were decreased and those of 134 lipids such as $\mathrm{PC}(38: 2)+\mathrm{H}, \mathrm{PS}(18: 1 / 24: 0)-\mathrm{H}$, and $\mathrm{DG}(18: 1 / 22: 1)+\mathrm{NH} 4$ were increased in the hippocampus in the EA group compared with the Sham group (Figure 6B). EA treatment increased 47 lipids such as $\mathrm{PC}(18: 0 \mathrm{p} / 20: 1)+\mathrm{HCOO}, \mathrm{MGDG}(10: 4 / 22: 6)+\mathrm{HCOO}$, and CerG1(d18:1/22:1)+H and decreased 192 lipids such as TG(18:1/18:1/18:1)+NH4, PS(42:7p)-H, and TG(17:0/18:1/18:1)+NH4 in the hippocampus in PTSD-treated mice (Figure 6C). Notably, 42 of the 241 lipid species in the hippocampus that were changed after PTSD treatment, such as Cer(d18:0+pO/24:0+O)+HCOO, PC(34:1)+H, and PS(20:4/22:6)-H, were normalized after EA treatment, suggesting that changes in these lipid species may be related to the biological effects of EA. Moreover, correlation analysis (Figure 6G) showed that the concentrations of 9 
lipids such as CerG1(d18:0/24:0+O)+H, $\mathrm{PE}(18: 0 / 18: 1)+\mathrm{H}$, and PS(44:11)-H in the hippocampus were negatively correlated with the time spent in the center squares in the OFT and in the open arms of the EPMT, but positively correlated with contextual and cued freezing time in the FCR. Concentrations of 23 lipids such as $\mathrm{CL}(18: 1 / 16: 0 / 16: 0 / 18: 1)-\mathrm{H}, \mathrm{PC}(34: 1)+\mathrm{H}$, and $\mathrm{SM}(\mathrm{d} 34: 1)+\mathrm{H}$ in the hippocampus were positively correlated with the time spent in the center squares in the OFT and in the open arms in the EPMT, but negatively correlated with contextual and cued freezing time in the FCR (see Table S4 for details), suggesting that changes in these lipid species in the hippocampus are related to the severity of PTSD.

Similar to the hippocampus, the PFC analysis revealed changes in the lipid concentrations in multiple species following mSPS and EA treatments (see Figure 6D-F and Table S5). Generally, the levels of 93 lipids such as PS(18:0/22:4)-H, PS(22:6/22:6)-H, and PS(42:7p)-H were decreased, and the levels of 37 lipids such as $\mathrm{PC}(32: 0)+\mathrm{H}, \mathrm{WE}(21: 1)+\mathrm{NH} 4$, and $\mathrm{TG}(16: 0 / 20: 4 / 20: 4)+\mathrm{NH} 4$ were increased in the PFC of PTSD-treated mice compared with Sham (Figure 6D). The concentrations of 30 lipids such as PS(38:4p)$H, P S(42: 7 p)-H$, and $P C(32: 0)+H$ were decreased and those of 201 lipids such as $P E(34: 2 p)-H$, $\mathrm{TG}(18: 3 / 18: 2 / 18: 2)+\mathrm{NH} 4$, and $\mathrm{PC}(33: 0)+\mathrm{H}$ were increased in EA-treated mice compared with Sham (Figure 6E). Moreover, EA treatment increased the levels of 11 lipids such as LPG(16:0)-H, $\mathrm{DG}(16: 0 / 18: 2)+\mathrm{NH} 4$, and $\mathrm{PG}(40: 5)+\mathrm{NH} 4$, and decreased the levels of 10 lipids such as PS(18:0/22:4)-H, LPE(20:2)-H, and LPE(20:3)-H compared with the PTSD group (Figure 6F). Notably, levels of 11 of the 130 lipid molecules in the PFC changed after mSPS such as $\operatorname{Cer}(\mathrm{d} 18: 0+\mathrm{pO} / 24: 0+0)+\mathrm{HCOO}, \mathrm{PC}(34: 1)+\mathrm{H}$, and PS(20:4/22:6)-H were normalized after EA treatment, suggesting that changes in these species in the PFC may be related to the biological effects of EA. Correlation analysis further showed that concentrations of LPG(16:0), DG(16:0/18:2)+NH4, PS(18:1/18:1)+H, LPG(20:4)-H, LPC(16:1p)+H, and PG(40:5)+NH4 in the PFC were negatively correlated, and concentrations of $L P E(20: 3)-H, L P E(20: 2)-H$, and DG(32:0e)+Na were positively correlated with contextual and cued freezing time in the FCR test (see Table S6 for details), suggesting that changes in these lipid species in the PFC are related to the severity of fear learning defects. Moreover, concentrations of DG(16:0/18:2)+NH4, PS(18:1/18:1)+H, and LPG(20:4)-H were positively correlated, and DG(32:0e)+Na was negatively correlated with the time spent in the open arms in the EPMT, suggesting that changes in these lipid species in the PFC are related to the severity of anxiety.

\section{Discussion}

In the present study, we performed comprehensive lipid profiling based on HPLC-MS/MS to assess the impact of EA treatment on lipidomic changes in the hippocampus and PFC of mice exposed to mSPS. We found that mSPS induced remarkable anxiety-like behaviors, fear-learning defects, and lipid changes in the brain, specifically in glycerolipids, glycerophospholipids, and sphingolipids. Moreover, the changes in lipid levels were more extensive in the hippocampus than in the PFC. Notably, early EA intervention attenuated mSPS-induced abnormal behaviors and partly normalized mSPS-induced lipid changes, notably in the hippocampus. Thus, a region-specific dysfunction of the brain lipidome may be involved in the pathogenesis of PTSD, and a region-specific regulation of the brain lipidome might partly account for the therapeutic effects of EA. 
In PTSD, the hippocampus and PFC are regions of central importance due to their prominent role in both the neuroendocrine stress response and memory alterations $[23,48]$. Atrophy of the hippocampus is one of the obvious pathologic changes in patients with PTSD and in animal models $[49,50]$. Hippocampal dysfunction might interact with traumatic experiences to influence the etiology and maintenance of PTSD $[51,52]$. Moreover, the amelioration of hippocampal structure and function was related to the alleviation of PTSD $[53,54]$. On the other hand, a clinical study found that youths with PTSD had sustained decreases in gray matter volume in the right ventromedial PFC (VmPFC) and bilateral ventrolateral PFC as well as decreased ventrolateral PFC-hippocampus connectivity over time [55]. Consistent with these results, PTSD remission has been associated with expansion of frontal pole surface area and an increase in vmPFC thickness over time [25]. Moreover, a preclinical study found that the protective effects of miR132 downregulation against behavioral impairment in rats exposed to single prolonged stress was related to a reduction in apoptosis in the PFC and an upregulation of brain-derived neurotrophic factor (BDNF) [56]. Notably, changes in the lipid composition of the hippocampus and PFC have been consistently associated with cognitive impairment and depressive-like behaviors $[47,57,58]$. Considering these results, we hypothesized that changes in the lipid composition of the hippocampus and PFC might also be associated with fear learning defects and anxiety-like behaviors in PTSD.

Glycerophospholipids and sphingolipids are important membrane lipid components that allow for the construction of a physical barrier $[39,59]$ and play crucial roles in regulating cell signaling [60]. Research has gradually revealed a general role for these components in neuropsychiatric diseases. Levels of PC and Cer are changed in the brain tissue of patients with bipolar disorder [61], and the relative abundances of $\mathrm{LPC}, \mathrm{PE}$, and $\mathrm{PI}$ in the plasma were positively correlated with the severity of depression $[62,63]$. Moreover, levels of Cer are elevated in patients with major depression and bipolar disorder [64], and concentrations of SM correlate with depression and anxiety symptoms [65]. Consistent with these observations, we found that mSPS induced significant changes in glycerophospholipid and sphingolipid levels in both the hippocampus and PFC. In the PTSD group, significant increases in hippocampal levels of LPS, LPC, PI, PE, Cer, and GM1 were observed, and in PFC, significant increases in levels of LPE and LPC were observed; significant decreases were observed in hippocampal CL, LPI, and SM and in prefrontal PS, LPG, CL, LPS, PA, and PG. Notably, changes in SM, Cer, GM1, PE, CL, and LPS in the hippocampus, which were correlated with anxiety or cognitive impairment, were alleviated after EA treatment. However, in the PFC, only changes in LPG and LPC were alleviated after EA treatment. SM constitutes the vast majority of cellular sphingolipid, and can be hydrolyzed to Cer by acid sphingomyelinase (ASM). Accumulating evidences suggest that the ASM/Cer system could potentially be a novel antidepressant target, and increased activity of the ASM/Cer system might be both an effect and a cause of immune system-mediated inflammation and dysregulation of oxidative stress [66, 67]. GM1 is a key factor in maintaining the mammalian neuronal functions and avoiding neurodegeneration $[68,69]$ and CL plays a role in the regulation of cellular energy metabolism and mitochondrial function [70]. LPC homeostasis is an endogenous mediator of myelin injury [71]; LPC levels are elevated in the cerebrospinal fluid of patients with multiple sclerosis [72] and in the brain following ischemia [73]. LPS and LPG represent an immunomodulatory effect $[74,75]$. PE is involved in the modulation of membrane fluidity 
and properties that protect the cell under conditions of oxidative stress [76]. Thus, mSPS-induced anxietylike behaviors and fear learning defects are related to an imbalance in levels of specific glycerophospholipids and sphingolipids in both the hippocampus and PFC. Lipidomic analyses revealed that 42 species, mainly belonging to the $C L, P C$, CerG1, PS, and SM series, were changed after mSPS in the hippocampus, while 11 species, mainly belong to the LPG and LPE series, were changed in the PFC, all of which were alleviated after EA. These results further indicate that the modulation of glycerophospholipid and sphingolipid levels after EA was brain area-specific, and that the lipid regulatory effect of EA was greater in the hippocampus than in the PFC.

In addition to the disturbance in levels of membrane lipid classes observed in the pathophysiology of PTSD, a growing body of evidence indicates that abnormalities in glycerolipid and fatty acyl compositions may also be involved $[40,77]$. The present study showed that mSPS increased the levels of glycerolipids in both the hippocampus (DG and TG) and the PFC (DG). However, only levels of TG, which were positively correlated with anxiety-like behaviors, were normalized after EA treatment, suggesting that the modulation of TG in the hippocampus is at least partially responsible for the protective effects of EA against PTSD.

In the brain, AcCa compounds functionally alter and stabilize membranes, thereby improving mitochondrial function and enhancing antioxidant activity [78, 79]. Recent studies have demonstrated that AcCa levels in the plasma of depressed subjects are lower than those of healthy controls and that these alterations correlate with the severity of the patients' depressive symptoms [80]. Our data show that mSPS induced a significant decrease in AcCa levels in both the hippocampus and PFC, which was negatively correlated with contextual freezing time and was normalized after EA treatment. Thus, a reduction in brain $\mathrm{AcCa}$ is involved in the pathophysiology of stress-induced fear learning defects and recovery of AcCa levels is associated with the protective effects of EA observed in this study.

The biophysical properties of lipids are influenced by the chain length (number of carbons) and the degree of saturation of the constituent fatty acyls [81]. Modulation of the composition of the fatty acyl chains and degree of saturation of membrane lipids can potentially affect neuronal functioning, at least in part through altered function of membrane-bound proteins $[58,82]$. The present study found that changes in the composition of fatty acyls in the hippocampus attributable to mSPS were ameliorated after EA treatment. However, the more extensive changes observed in the PFC were not normalized after EA treatment, suggesting that fatty acyl composition in the PFC is more vulnerable to mSPS and does not recover as quickly as that in the hippocampus. Notably, we also found that after mSPS, the concentration of Co(Q9) was lowered in both the hippocampus and PFC and was effectively normalized after EA treatment. $\mathrm{CoQ}$ is a redox-active molecule that plays a fundamental role in mitochondrial energy generation and functions as a potent endogenous antioxidant [83]. Our results indicate that a disturbance of the brain antioxidant system is involved in the pathogenesis of PTSD and that $\mathrm{Co}(\mathrm{Q} 9)+\mathrm{NH} 4$ is potentially a molecular target for the treatment of PTSD. 
Recent work has previously reported an influence of acupuncture treatment on both depressive-like behaviors and the lipidomics of the liver [84]. However, the neuroprotective effects of EA are closely related to its action parameters and action time, and precedents exist for suspecting that early intervention using EA might be an effective strategy for preventing the development of PTSD symptoms $[54,85]$. Previous studies have found that EA stimulation $(2 / 15 \mathrm{~Hz})$ applied at the GV20 ("Bai hui") point enhanced motor performance recovery and BDNF expression in rats with cerebral infarction [86]. EA treatment has also been shown to reduce glutamate toxicity and exert antiapoptotic effects in a rat stroke model $[87,88]$. Importantly, our previous work found that early EA intervention applied at the GV20 point with the same frequencies $(2 / 15 \mathrm{~Hz})$ prevents PTSD-like behaviors in a rat model of PTSD [28].

Consistent with these results, the present study indicates that early intervention using EA administered for seven continuous days exerts protective effects on experimental PTSD in mice and mobilizes changes in brain lipid compositions, mainly in the hippocampus. Thus, we speculate that different frequencies and current intensity of EA might induce different neuromodulation effects by influence the composition of lipids in the brain. Neither did we investigate the effects of EA at different time points, nor the relationships between different parameters of EA and the composition of lipids in the brain, which should be the goals of future research.

Taken together, our results indicate that lipidomic changes in the PFC and hippocampus may be a key aspect of the pathogenesis of PTSD. Importantly, the functions of these lipids are mainly focused on antiinflammation, energy metabolism, oxidative stress, and neuroprotection, which may partly explain the neuroprotective effect of EA. The observation of a correlation between levels of oxidative stress, inflammation, or levels of neurotrophic factors in the hippocampus or PFC might provide further insight into this hypothesis. The present study found that levels of $\mathrm{AcCa}, \mathrm{CL}$, and Co were decreased after $\mathrm{EA}$ while that of LPC was increased in both hippocampus and PFC. However, changes in LPS and DG showed an opposite trend in the hippocampus and PFC, which might relate to the divergent structures and functions of these brain regions. The precise mechanisms of brain area-specific modulation after EA and the effects of specific lipid species on PTSD-like behaviors require further investigation. Furthermore, although all mice in this study were exposed to isoflurane inhalation anesthesia, we cannot rule out an anesthetic effect on our results due to the regulatory effect of isoflurane on brain lipidomics and its potential influence on brain oxidative stress, inflammation, and levels of neurotrophic factors [89-91].

In summary, our study revealed distinct changes in lipid composition within the hippocampus and PFC in mice after exposure to mSPS. We further confirmed that the hippocampus is more sensitive to mSPSinduced lipid modulation than is the PFC and showed that EA is effective in normalizing the changes in lipid composition in the hippocampus, notably changes in sphingolipids and glycerophospholipids. Based on these observations, we conclude that the distribution of lipids across the hippocampus and PFC may dictate regional susceptibility to stress and explain the neuromodulatory effects of EA. However, the influence of behavioral testing and different EA parameters on brain lipids, as well as the precise mechanism through which EA regulates lipid composition, require further investigation. 


\section{Abbreviations}

PTSD: posttraumatic stress disorder; EA: electroacupuncture; PFC: prefrontal cortex; mSPS: modified single prolonged stress; HPLC-MS/MS: high performance liquid chromatography-tandem mass spectrometry; OFT: open field test; EPMT: elevated-plus maze test; FCR: fear conditioning response test; MTBE: methyl tert-butyl ether; SD: standard deviation; ANOVA: one-way analysis of variance; DG: diglyceride; TG: triglyceride; SM: sphingomyelin; Cer: ceramides; GM1: gangliosides; CL: cardiolipin; LPG: lysophosphatidylglycerol; LPI: lysophosphatidylinositol; LPS: Iysophosphatidylserine; PA: phosphatidic acid; PE: phosphatidylethanolamine; PG: phosphatidylglycerol; PI: phosphatidylinositol; AcCa: acylcarnitine; SQDG: sulfoquinovosyldiacylglycerol; MGDG: monogalactosyldiacylglycerol; Co: coenzyme; So: sphingosine; FA: fatty acid; DGDG: digalactosyldiacylglycerol; LPC: Iysophosphatidylcholine; PC: phosphatidylcholine; WE: wax esters; CerG2GNAc1: simple Glc series; phSM: sphingomyelin; PS: phosphatidylserine; CerG1: glucosylceramides; ASM: acid sphingomyelinase; NSM: neutral sphingomyelinase

\section{Declarations}

\section{Acknowledgements}

We would like to thank Shanghai Applied Protein Technology Co., Ltd., for technological assistance. This study was funded by the National Natural Science Foundation of China [grant numbers 81904280 , 81974215, 82101594, and 82171512].

\section{Code Availability}

Not applicable

\section{Authors contributions}

$\mathrm{CZ}, \mathrm{FX}, \mathrm{QS}, \mathrm{SX}, \mathrm{TZ}, \mathrm{XM}, \mathrm{LY}$ and $\mathrm{CL}$ were responsible for collecting the data and behavioral evaluation. ZP and HW financed and designed the study and supervised the data collection and analysis, $\mathrm{CZ}$ analyzed the data and wrote the first draft with ZP and FX. All other authors provided data, reviewed the results, and contributed to the final draft of the report.

\section{Funding}

This study was funded by the National Natural Science Foundation of China [grant numbers 81904280 , 81974215, 82101594, and 82171512].

\section{Data availability}

All data generated or analysed during this study are included in this published article [and its supplementary information files]. 


\section{Ethics Approval}

The experimental procedures of this study were in accordance with the National Institutes of Health Guide for the Care and Use of Laboratory Animals and were approved by the Animal Use and Protection Committee of the Fourth Military Medical University (KY20193003).

\section{Consent to Participate}

Not applicable

\section{Consent of Publication}

All authors read and approved the final manuscript.

\section{Conflict of Interest}

The authors have declared that no competing interest exists

\section{References}

1. Steenkamp MM, Litz BT, Hoge CW, and Marmar CR (2015) Psychotherapy for Military-Related PTSD: A Review of Randomized Clinical Trials. JAMA 314: 489-500. http://dio.10.1001/jama.2015.8370

2. O'Donovan A, Ahmadian AJ, Neylan TC, Pacult MA, Edmondson D, and Cohen BE (2017) Current posttraumatic stress disorder and exaggerated threat sensitivity associated with elevated inflammation in the Mind Your Heart Study. Brain Behav Immun 60: 198-205. http://dio.10.1016/j.bbi.2016.10.014

3. Careaga MBL, Girardi CEN, and Suchecki D (2016) Understanding posttraumatic stress disorder through fear conditioning, extinction and reconsolidation. Neurosci Biobehav Rev 71: 48-57. http://dio.10.1016/j.neubiorev.2016.08.023

4. Mazza MG, De Lorenzo R, Conte C, Poletti S, Vai B, Bollettini I, et al (2020) Anxiety and depression in COVID-19 survivors: Role of inflammatory and clinical predictors. Brain Behav Immun 89: 594-600. http://dio.10.1016/j.bbi.2020.07.037

5. Guessoum SB, Lachal J, Radjack R, Carretier E, Minassian S, Benoit L, et al (2020) Adolescent psychiatric disorders during the COVID-19 pandemic and lockdown. Psychiatry Res 291: 113264. http://dio.10.1016/j.psychres.2020.113264

6. Tang W, Hu T, Hu B, Jin C, Wang G, Xie C, et al (2020) Prevalence and correlates of PTSD and depressive symptoms one month after the outbreak of the COVID-19 epidemic in a sample of homequarantined Chinese university students. J Affect Disord 274: 1-7. http://dio.10.1016/j.jad.2020.05.009

7. Brenner L, Kollner V, and Bachem R (2019) Symptom burden and work-related impairment among patients with PTSD and complex PTSD. Eur J Psychotraumatol 10: 1694766. 
http://dio.10.1080/20008198.2019.1694766

8. Nichter B, Norman S, Haller M, and Pietrzak RH (2019) Psychological burden of PTSD, depression, and their comorbidity in the U.S. veteran population: Suicidality, functioning, and service utilization. J Affect Disord 256: 633-640. http://dio.10.1016/j.jad.2019.06.072

9. Jeffreys M, Capehart B, and Friedman MJ (2012) Pharmacotherapy for posttraumatic stress disorder: review with clinical applications. J Rehabil Res Dev 49: 703-15.

10. Mello PG, Silva GR, Donat JC, and Kristensen CH (2013) An update on the efficacy of cognitivebehavioral therapy, cognitive therapy, and exposure therapy for posttraumatic stress disorder. Int $J$ Psychiatry Med 46: 339-57. http://dio.10.2190/PM.46.4.b

11. Krystal JH, Davis LL, Neylan TC, M AR, Schnurr PP, Stein MB, et al (2017) It Is Time to Address the Crisis in the Pharmacotherapy of Posttraumatic Stress Disorder: A Consensus Statement of the PTSD Psychopharmacology Working Group. Biol Psychiatry 82: e51-e59. http://dio.10.1016/j.biopsych.2017.03.007

12. Fenster RJ, Lebois LAM, Ressler KJ, and Suh J (2018) Brain circuit dysfunction in post-traumatic stress disorder: from mouse to man. Nat Rev Neurosci 19: 535-551. http://dio.10.1038/s41583-0180039-7

13. Langevin HM, Wayne PM, Macpherson H, Schnyer R, Milley RM, Napadow V, et al (2011) Paradoxes in acupuncture research: strategies for moving forward. Evid Based Complement Alternat Med 2011: 180805. http://dio.10.1155/2011/180805

14. Zhang R, Lao L, Ren K, and Berman BM (2014) Mechanisms of acupuncture-electroacupuncture on persistent pain. Anesthesiology 120: 482-503. http://dio.10.1097/ALN.0000000000000101

15. Liu Z, Liu Y, Xu H, He L, Chen Y, Fu L, et al (2017) Effect of Electroacupuncture on Urinary Leakage Among Women With Stress Urinary Incontinence: A Randomized Clinical Trial. JAMA 317: 24932501. http://dio.10.1001/jama.2017.7220

16. Chen JDZ, Ni M, and Yin J (2018) Electroacupuncture treatments for gut motility disorders. Neurogastroenterol Motil 30: e13393. http://dio.10.1111/nmo.13393

17. Tamtaji OR, Naderi Taheri M, Notghi F, Alipoor R, Bouzari R, and Asemi Z (2019) The effects of acupuncture and electroacupuncture on Parkinson's disease: Current status and future perspectives for molecular mechanisms. J Cell Biochem 120: 12156-12166. http://dio.10.1002/jcb.28654

18. Wang Z, Dong H, Wang Q, Zhang L, Wu X, Zhou Z, et al (2019) Effects of electroacupuncture on anxiety and depression in unmarried patients with polycystic ovarian syndrome: secondary analysis of a pilot randomised controlled trial. Acupunct Med 37: 40-46. http://dio.10.1136/acupmed-2017011615

19. Li X, Dai Q, Shi Z, Chen H, Hu Y, Wang X, et al (2019) Clinical Efficacy and Safety of Electroacupuncture in Migraine Treatment: A Systematic Review and Network Meta-Analysis. Am J Chin Med 47: 1755-1780. http://dio.10.1142/S0192415X19500897

20. He J, Zhao C, Liu W, Huang J, Liang S, Chen L, et al (2018) Neurochemical changes in the hippocampus and prefrontal cortex associated with electroacupuncture for learning and memory 
impairment. Int J Mol Med 41: 709-716. http://dio.10.3892/ijmm.2017.3287

21. Chen L, Gong XK, Leng CL, Ma BM, Ru Q, Xiong Q, et al (2019) 2Hz-electroacupuncture attenuates heroin-seeking behaviors via adjusts CB1-Rs and CB2-Rs expression in relapse-relevant brain regions of heroin self-administration rats. Physiol Res 68: 835-844. http://dio.10.33549/physiolres.934106

22. Marcus DJ, Bedse G, Gaulden AD, Ryan JD, Kondev V, Winters ND, et al (2020) Endocannabinoid Signaling Collapse Mediates Stress-Induced Amygdalo-Cortical Strengthening. Neuron 105: 10621076 e6. http://dio.10.1016/j.neuron.2019.12.024

23. Smith KL, Kassem MS, Clarke DJ, Kuligowski MP, Bedoya-Perez MA, Todd SM, et al (2019) Microglial cell hyper-ramification and neuronal dendritic spine loss in the hippocampus and medial prefrontal cortex in a mouse model of PTSD. Brain Behav Immun 80: 889-899.

http://dio.10.1016/j.bbi.2019.05.042

24. Lambert HK and McLaughlin KA (2019) Impaired hippocampus-dependent associative learning as a mechanism underlying PTSD: A meta-analysis. Neurosci Biobehav Rev 107: 729-749. http://dio.10.1016/j.neubiorev.2019.09.024

25. Heyn SA and Herringa RJ (2019) Longitudinal cortical markers of persistence and remission of pediatric PTSD. Neuroimage Clin 24: 102028. http://dio.10.1016/j.nicl.2019.102028

26. Wang Y, Hu YP, Wang WC, Pang RZ, and Zhang AR (2012) Clinical studies on treatment of earthquake-caused posttraumatic stress disorder using electroacupuncture. Evid Based Complement Alternat Med 2012: 431279. http://dio.10.1155/2012/431279

27. Li M, Xie Y, Niu K, and Li K (2020) Electroacupuncture ameliorates post-traumatic stress disorder in rats via a mechanism involving the BDNF-TrkB signaling pathway. Cell Mol Biol (Noisy-le-grand) 66: 165-170.

28. Xue F, Xue SS, Liu L, Sang HF, Ma QR, Tan QR, et al (2019) Early intervention with electroacupuncture prevents PTSD-like behaviors in rats through enhancing hippocampal endocannabinoid signaling. Prog Neuropsychopharmacol Biol Psychiatry 93: 171-181. http://dio.10.1016/j.pnpbp.2019.03.018

29. Zhou CH, Xue F, Xue SS, Sang HF, Liu L, Wang Y, et al (2019) Electroacupuncture Pretreatment Ameliorates PTSD-Like Behaviors in Rats by Enhancing Hippocampal Neurogenesis via the Keap1/Nrf2 Antioxidant Signaling Pathway. Front Cell Neurosci 13: 275. http://dio.10.3389/fncel.2019.00275

30. Hashimoto M, Katakura M, Tanabe Y, Al Mamun A, Inoue T, Hossain S, et al (2015) n-3 fatty acids effectively improve the reference memory-related learning ability associated with increased brain docosahexaenoic acid-derived docosanoids in aged rats. Biochim Biophys Acta 1851: 203-9. http://dio.10.1016/j.bbalip.2014.10.009

31. McDougall M, Choi J, Magnusson K, Truong L, Tanguay R, and Traber MG (2017) Chronic vitamin E deficiency impairs cognitive function in adult zebrafish via dysregulation of brain lipids and energy metabolism. Free Radic Biol Med 112: 308-317. http://dio.10.1016/j.freeradbiomed.2017.08.002

32. Hussain G, Wang J, Rasul A, Anwar H, Imran A, Qasim M, et al (2019) Role of cholesterol and sphingolipids in brain development and neurological diseases. Lipids Health Dis 18: 26. 
http://dio.10.1186/s12944-019-0965-z

33. Araque A, Castillo PE, Manzoni OJ, and Tonini R (2017) Synaptic functions of endocannabinoid signaling in health and disease. Neuropharmacology 124: 13-24.

http://dio.10.1016/j.neuropharm.2017.06.017

34. Egawa J, Pearn ML, Lemkuil BP, Patel PM, and Head BP (2016) Membrane lipid rafts and neurobiology: age-related changes in membrane lipids and loss of neuronal function. J Physiol 594: 4565-79. http://dio.10.1113/JP270590

35. Wu L, Zhang X, and Zhao L (2018) Human ApoE Isoforms Differentially Modulate Brain Glucose and Ketone Body Metabolism: Implications for Alzheimer's Disease Risk Reduction and Early Intervention. J Neurosci 38: 6665-6681. http://dio.10.1523/JNEUROSCI.2262-17.2018

36. Scola G, McNamara RK, Croarkin PE, Leffler JM, Cullen KR, Geske JR, et al (2016) Lipid peroxidation biomarkers in adolescents with or at high-risk for bipolar disorder. J Affect Disord 192: 176-83. http://dio.10.1016/j.jad.2015.12.020

37. Bartoli F, Di Brita C, Crocamo C, Clerici M, and Carra G (2017) Lipid profile and suicide attempt in bipolar disorder: A meta-analysis of published and unpublished data. Prog Neuropsychopharmacol Biol Psychiatry 79: 90-95. http://dio.10.1016/j.pnpbp.2017.06.008

38. Kavoor AR, Mitra S, Kumar S, Sisodia AK, and Jain R (2017) Lipids, aggression, suicidality and impulsivity in drug-naive/drug-free patients of schizophrenia. Asian J Psychiatr 27: 129-136. http://dio.10.1016/j.ajp.2017.03.002

39. Muller CP, Reichel M, Muhle C, Rhein C, Gulbins E, and Kornhuber J (2015) Brain membrane lipids in major depression and anxiety disorders. Biochim Biophys Acta 1851: 1052-65. http://dio.10.1016/j.bbalip.2014.12.014

40. Huguenard CJC, Cseresznye A, Evans JE, Oberlin S, Langlois H, Ferguson S, et al (2020) Plasma Lipidomic Analyses in Cohorts With mTBI and/or PTSD Reveal Lipids Differentially Associated With Diagnosis and APOE epsilon4 Carrier Status. Front Physiol 11: 12. http://dio.10.3389/fphys.2020.00012

41. Wang W, Liu Y, Zheng H, Wang HN, Jin X, Chen YC, et al (2008) A modified single-prolonged stress model for post-traumatic stress disorder. Neurosci Lett 441: 237-41. http://dio.10.1016/j.neulet.2008.06.031

42. Feng DY, Guo BL, Liu GH, Xu K, Yang J, Tao K, et al (2020) Nerve growth factor against PTSD symptoms: Preventing the impaired hippocampal cytoarchitectures. Prog Neurobiol 184: 101721. http://dio.10.1016/j.pneurobio.2019.101721

43. Geng W, Cai L, Han K, Li D, Mo Y, Dai Q, et al (2020) Electroacupuncture Pretreatment Alleviates Cerebral Ischemia-Reperfusion Injury by Increasing GSK-3beta Phosphorylation Level via Adenosine A1 Receptor. Biomed Res Int 2020: 6848450. http://dio.10.1155/2020/6848450

44. Sullivan GM, Apergis J, Gorman JM, and LeDoux JE (2003) Rodent doxapram model of panic: behavioral effects and c-Fos immunoreactivity in the amygdala. Biol Psychiatry 53: 863-70. http://dio.10.1016/s0006-3223(02)01733-x 
45. Liu WZ, Zhang WH, Zheng ZH, Zou JX, Liu XX, Huang SH, et al (2020) Identification of a prefrontal cortex-to-amygdala pathway for chronic stress-induced anxiety. Nat Commun 11: 2221. http://dio.10.1038/s41467-020-15920-7

46. Jiang L, Gu H, Lin Y, Xu W, Zhu R, Kong J, et al (2017) Remodeling of brain lipidome in methamphetamine-sensitized mice. Toxicol Lett 279: 67-76. http://dio.10.1016/j.toxlet.2017.07.214

47. Xue SS, Zhou CH, Xue F, Liu L, Cai YH, Luo JF, et al (2020) The impact of repetitive transcranial magnetic stimulation and fluoxetine on the brain lipidome in a rat model of chronic unpredictable stress. Prog Neuropsychopharmacol Biol Psychiatry 102: 109946. http://dio.10.1016/j.pnpbp.2020.109946

48. Harnett NG, Goodman AM, and Knight DC (2020) PTSD-related neuroimaging abnormalities in brain function, structure, and biochemistry. Exp Neurol 330: 113331. http://dio.10.1016/j.expneurol.2020.113331

49. Lindauer RJ, Olff M, van Meijel EP, Carlier IV, and Gersons BP (2006) Cortisol, learning, memory, and attention in relation to smaller hippocampal volume in police officers with posttraumatic stress disorder. Biol Psychiatry 59: 171-7. http://dio.10.1016/j.biopsych.2005.06.033

50. Kikuchi A, Shimizu K, Nibuya M, Hiramoto T, Kanda Y, Tanaka T, et al (2008) Relationship between post-traumatic stress disorder-like behavior and reduction of hippocampal 5-bromo-2'-deoxyuridinepositive cells after inescapable shock in rats. Psychiatry Clin Neurosci 62: 713-20. http://dio.10.1111/j.1440-1819.2008.01875.x

51. Acheson DT, Gresack JE, and Risbrough VB (2012) Hippocampal dysfunction effects on context memory: possible etiology for posttraumatic stress disorder. Neuropharmacology 62: 674-85. http://dio.10.1016/j.neuropharm.2011.04.029

52. Bae S, Sheth C, Legarreta M, McGlade E, Lyoo IK, and Yurgelun-Todd DA (2020) Volume and shape analysis of the Hippocampus and amygdala in veterans with traumatic brain injury and posttraumatic stress disorder. Brain Imaging Behav 14: 1850-1864. http://dio.10.1007/s11682-01900127-2

53. Nie PY, Tong L, Li MD, Fu CH, Peng JB, and Ji LL (2021) miR-142 downregulation alleviates rat PTSDlike behaviors, reduces the level of inflammatory cytokine expression and apoptosis in hippocampus, and upregulates the expression of fragile $X$ mental retardation protein. $J$ Neuroinflammation 18: 17. http://dio.10.1186/s12974-020-02064-0

54. Nie H, Peng Z, Lao N, Wang H, Chen Y, Fang Z, et al (2014) Rosmarinic acid ameliorates PTSD-like symptoms in a rat model and promotes cell proliferation in the hippocampus. Prog Neuropsychopharmacol Biol Psychiatry 51: 16-22. http://dio.10.1016/j.pnpbp.2014.01.002

55. Heyn SA, Keding TJ, Ross MC, Cisler JM, Mumford JA, and Herringa RJ (2019) Abnormal Prefrontal Development in Pediatric Posttraumatic Stress Disorder: A Longitudinal Structural and Functional Magnetic Resonance Imaging Study. Biol Psychiatry Cogn Neurosci Neuroimaging 4: 171-179. http://dio.10.1016/j.bpsc.2018.07.013 
56. Tong L, Li MD, Nie PY, Chen Y, Chen YL, and Ji LL (2021) miR-132 downregulation alleviates behavioral impairment of rats exposed to single prolonged stress, reduces the level of apoptosis in PFC, and upregulates the expression of MeCP2 and BDNF. Neurobiol Stress 14: 100311. http://dio.10.1016/j.ynstr.2021.100311

57. Zhou C, Cai M, Wang Y, Wu W, Yin Y, Wang X, et al (2021) The Effects of Repetitive Transcranial Magnetic Stimulation on Cognitive Impairment and the Brain Lipidome in a Cuprizone-Induced Mouse Model of Demyelination. Front Neurosci 15: 706786. http://dio.10.3389/fnins.2021.706786

58. Oliveira TG, Chan RB, Bravo FV, Miranda A, Silva RR, Zhou B, et al (2016) The impact of chronic stress on the rat brain lipidome. Mol Psychiatry 21: 80-8. http://dio.10.1038/mp.2015.14

59. Muallem S, Chung WY, Jha A, and Ahuja M (2017) Lipids at membrane contact sites: cell signaling and ion transport. EMBO Rep 18: 1893-1904. http://dio.10.15252/embr.201744331

60. Nadler A, Yushchenko DA, Muller R, Stein F, Feng S, Mulle C, et al (2015) Exclusive photorelease of signalling lipids at the plasma membrane. Nat Commun 6: 10056. http://dio.10.1038/ncomms10056

61. Schwarz E, Prabakaran S, Whitfield P, Major H, Leweke FM, Koethe D, et al (2008) High throughput lipidomic profiling of schizophrenia and bipolar disorder brain tissue reveals alterations of free fatty acids, phosphatidylcholines, and ceramides. J Proteome Res 7: 4266-77. http://dio.10.1021/pr800188y

62. Liu X, Li J, Zheng P, Zhao X, Zhou C, Hu C, et al (2016) Plasma lipidomics reveals potential lipid markers of major depressive disorder. Anal Bioanal Chem 408: 6497-507. http://dio.10.1007/s00216016-9768-5

63. Ogawa S, Hattori K, Ota M, Hidese S, Miyakawa T, Matsumura R, et al (2020) Altered ethanolamine plasmalogen and phosphatidylethanolamine levels in blood plasma of patients with bipolar disorder. Psychiatry Clin Neurosci 74: 204-210. http://dio.10.1111/pcn.12967

64. Brunkhorst-Kanaan N, Klatt-Schreiner K, Hackel J, Schroter K, Trautmann S, Hahnefeld L, et al (2019) Targeted lipidomics reveal derangement of ceramides in major depression and bipolar disorder. Metabolism 95: 65-76. http://dio.10.1016/j.metabol.2019.04.002

65. Demirkan A, Isaacs A, Ugocsai P, Liebisch G, Struchalin M, Rudan I, et al (2013) Plasma phosphatidylcholine and sphingomyelin concentrations are associated with depression and anxiety symptoms in a Dutch family-based lipidomics study. J Psychiatr Res 47: 357-62. http://dio.10.1016/j.jpsychires.2012.11.001

66. Kornhuber J, Muller CP, Becker KA, Reichel M, and Gulbins E (2014) The ceramide system as a novel antidepressant target. Trends Pharmacol Sci 35: 293-304. http://dio.10.1016/j.tips.2014.04.003

67. Kurz J, Parnham MJ, Geisslinger G, and Schiffmann S (2019) Ceramides as Novel Disease Biomarkers. Trends Mol Med 25: 20-32. http://dio.10.1016/j.molmed.2018.10.009

68. Chiricozzi E, Lunghi G, Di Biase E, Fazzari M, Sonnino S, and Mauri L (2020) GM1 Ganglioside Is A Key Factor in Maintaining the Mammalian Neuronal Functions Avoiding Neurodegeneration. Int $\mathrm{J}$ Mol Sci 21. http://dio.10.3390/ijms21030868 
69. Meng C, Yao XQ, Chang RJ, Wang SL, Wang X, Ma DQ, et al (2020) Exogenous GM1 Ganglioside Attenuates Ketamine-Induced Neurocognitive Impairment in the Developing Rat Brain. Anesth Analg 130: 505-517. http://dio.10.1213/ANE.0000000000004570

70. Acaz-Fonseca E, Ortiz-Rodriguez A, Garcia-Segura LM, and Astiz M (2020) Sex differences and gonadal hormone regulation of brain cardiolipin, a key mitochondrial phospholipid. J Neuroendocrinol 32: e12774. http://dio.10.1111/jne.12774

71. Plemel JR, Michaels NJ, Weishaupt N, Caprariello AV, Keough MB, Rogers JA, et al (2018) Mechanisms of lysophosphatidylcholine-induced demyelination: A primary lipid disrupting myelinopathy. Glia 66: 327-347. http://dio.10.1002/glia.23245

72. Shanta SR, Choi CS, Lee JH, Shin CY, Kim YJ, Kim KH, et al (2012) Global changes in phospholipids identified by MALDI MS in rats with focal cerebral ischemia. J Lipid Res 53: 1823-31. http://dio.10.1194/jlr.M022558

73. Pieragostino D, D'Alessandro M, di loia M, Rossi C, Zucchelli M, Urbani A, et al (2015) An integrated metabolomics approach for the research of new cerebrospinal fluid biomarkers of multiple sclerosis. Mol Biosyst 11: 1563-72. http://dio.10.1039/c4mb00700j

74. Ogasawara D, Ichu TA, Vartabedian VF, Benthuysen J, Jing H, Reed A, et al (2018) Selective blockade of the lyso-PS lipase ABHD12 stimulates immune responses in vivo. Nat Chem Biol 14: 1099-1108. http://dio.10.1038/s41589-018-0155-8

75. Favila MA, Geraci NS, Jayakumar A, Hickerson S, Mostrom J, Turco SJ, et al (2015) Differential Impact of LPG-and PG-Deficient Leishmania major Mutants on the Immune Response of Human Dendritic Cells. PLoS Negl Trop Dis 9: e0004238. http://dio.10.1371/journal.pntd.0004238

76. Wallner S, Orso E, Grandl M, Konovalova T, Liebisch G, and Schmitz G (2018) Phosphatidylcholine and phosphatidylethanolamine plasmalogens in lipid loaded human macrophages. PLoS One 13: e0205706. http://dio.10.1371/journal.pone.0205706

77. de Vries GJ, Mocking R, Lok A, Assies J, Schene A, and Olff M (2016) Fatty acid concentrations in patients with posttraumatic stress disorder compared to healthy controls. J Affect Disord 205: 351359. http://dio.10.1016/j.jad.2016.08.021

78. Tarasenko TN, Cusmano-Ozog K, and McGuire PJ (2018) Tissue acylcarnitine status in a mouse model of mitochondrial beta-oxidation deficiency during metabolic decompensation due to influenza virus infection. Mol Genet Metab 125: 144-152. http://dio.10.1016/j.ymgme.2018.06.012

79. Rutkowsky JM, Knotts TA, Ono-Moore KD, McCoin CS, Huang S, Schneider D, et al (2014) Acylcarnitines activate proinflammatory signaling pathways. Am J Physiol Endocrinol Metab 306: E1378-87. http://dio.10.1152/ajpendo.00656.2013

80. Cassol E, Misra V, Morgello S, Kirk GD, Mehta SH, and Gabuzda D (2015) Altered Monoamine and Acylcarnitine Metabolites in HIV-Positive and HIV-Negative Subjects With Depression. J Acquir Immune Defic Syndr 69: 18-28. http://dio.10.1097/QAl.0000000000000551

81. Guha M, Gantz DL, and Gursky O (2008) Effects of acyl chain length, unsaturation, and pH on thermal stability of model discoidal HDLs. J Lipid Res 49: 1752-61. http://dio.10.1194/jlr.M800106- 
JLR200

82. Carta M, Lanore F, Rebola N, Szabo Z, Da Silva SV, Lourenco J, et al (2014) Membrane lipids tune synaptic transmission by direct modulation of presynaptic potassium channels. Neuron 81: 787-99. http://dio.10.1016/j.neuron.2013.12.028

83. Pandey R, Riley CL, Mills EM, and Tiziani S (2018) Highly sensitive and selective determination of redox states of coenzymes Q9 and Q10 in mice tissues: Application of orbitrap mass spectrometry. Anal Chim Acta 1011: 68-76. http://dio.10.1016/j.aca.2018.01.066

84. Jung J, Lee SM, Lee MJ, Ryu JS, Song JH, Lee JE, et al (2021) Lipidomics reveals that acupuncture modulates the lipid metabolism and inflammatory interaction in a mouse model of depression. Brain Behav Immun 94: 424-436. http://dio.10.1016/j.bbi.2021.02.003

85. Peng Z, Zhang R, Wang H, Chen Y, Xue F, Wang L, et al (2013) Ziprasidone ameliorates anxiety-like behaviors in a rat model of PTSD and up-regulates neurogenesis in the hippocampus and hippocampus-derived neural stem cells. Behav Brain Res 244: 1-8.

http://dio.10.1016/j.bbr.2013.01.032

86. Kim MW, Chung YC, Jung HC, Park MS, Han YM, Chung YA, et al (2012) Electroacupuncture enhances motor recovery performance with brain-derived neurotrophic factor expression in rats with cerebral infarction. Acupunct Med 30: 222-6. http://dio.10.1136/acupmed-2011-010126

87. Zhu X, Yin J, Li L, Ma L, Tan H, Deng J, et al (2013) Electroacupuncture preconditioning-induced neuroprotection may be mediated by glutamate transporter type 2. Neurochem Int 63: 302-8. http://dio.10.1016/j.neuint.2013.06.017

88. Zhou H, Zhang Z, Wei H, Wang F, Guo F, Gao Z, et al (2013) Activation of STAT3 is involved in neuroprotection by electroacupuncture pretreatment via cannabinoid CB1 receptors in rats. Brain Res 1529: 154-64. http://dio.10.1016/j.brainres.2013.07.006

89. Lee YM, Song BC, and Yeum KJ (2015) Impact of Volatile Anesthetics on Oxidative Stress and Inflammation. Biomed Res Int 2015: 242709. http://dio.10.1155/2015/242709

90. Miao HH, Zhang Y, Ding GN, Hong FX, Dong P, and Tian M (2017) Ginsenoside Rb1 Attenuates Isoflurane/surgery-induced Cognitive Dysfunction via Inhibiting Neuroinflammation and Oxidative Stress. Biomed Environ Sci 30: 363-372. http://dio.10.3967/bes2017.047

91. Zhou $\mathrm{CH}$, Zhang $\mathrm{YH}$, Xue F, Xue SS, Chen YC, Gu T, et al (2017) Isoflurane exposure regulates the cell viability and BDNF expression of astrocytes via upregulation of TREK1. Mol Med Rep 16: 7305-7314. http://dio.10.3892/mmr.2017.7547

\section{Figures}


$\mathbf{A}$

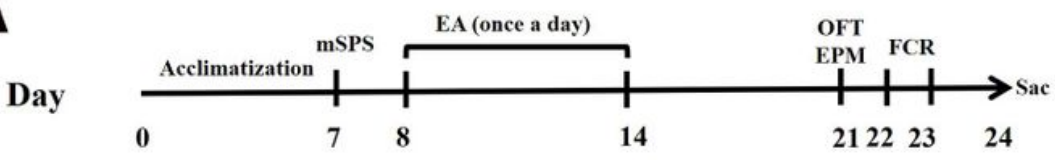

\begin{tabular}{l|l|l|}
\cline { 2 - 3 } Sham & False stimulation \\
\hline
\end{tabular}

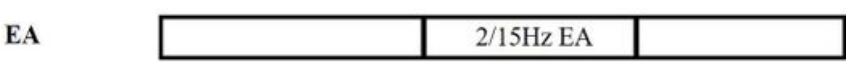

PTSD

PTSD + EA

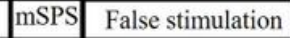

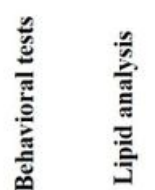

\begin{tabular}{|l|l|}
\hline $\mathrm{mSPS}$ & $2 / 15 \mathrm{~Hz} \mathrm{EA}$ \\
\hline
\end{tabular}
B

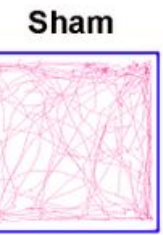

PTSD

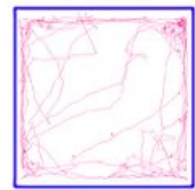

PTSD+EA

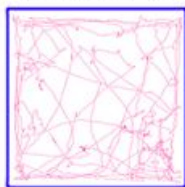

$\mathbf{E}$

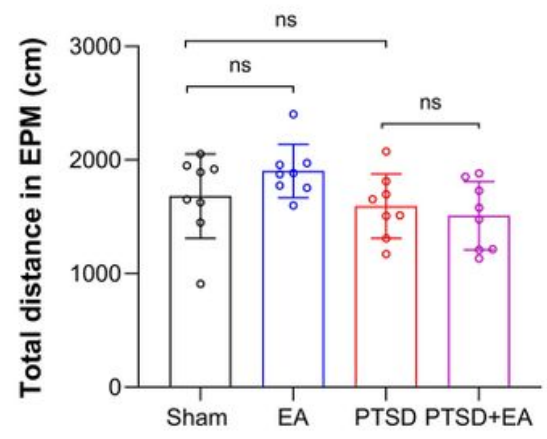

H

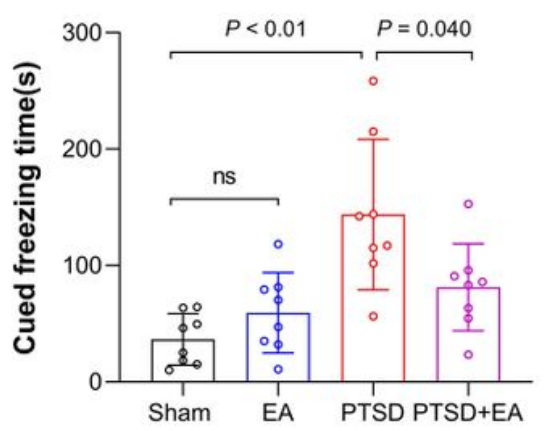

Figure 1

Early intervention using electroacupuncture (EA) ameliorates posttraumatic stress disorder (PTSD)-like behaviors in model mice treated with a modified single prolonged stress (mSPS) procedure. (A) The experimental design. After one week of adaptation, animals were administered mSPS or a sham treatment, then $\mathrm{EA}(2 / 15 \mathrm{~Hz}, 1.0 \mathrm{~mA})$ or false stimulation was administered once a day for 30 min per day from days 8 to 14. Behavioral alterations were assessed from days 21 to 23 , then mice were sacrificed (sac) for tissue collection. (B) Representative real-time movement traces in the open field test (OFT) for each group. (C) Quantification of the total distance traveled during the OFT. (D) Time spent in the center squares in the OFT. (E) Quantification of the total distance traveled in the elevated-plus maze test 
(EPMT). (F) Time spent in the open arms in the EPMT. (G) Freezing time measured in the contextual fear response test. $(H)$ Freezing time measured in the cued fear response test.

A $\quad$ B
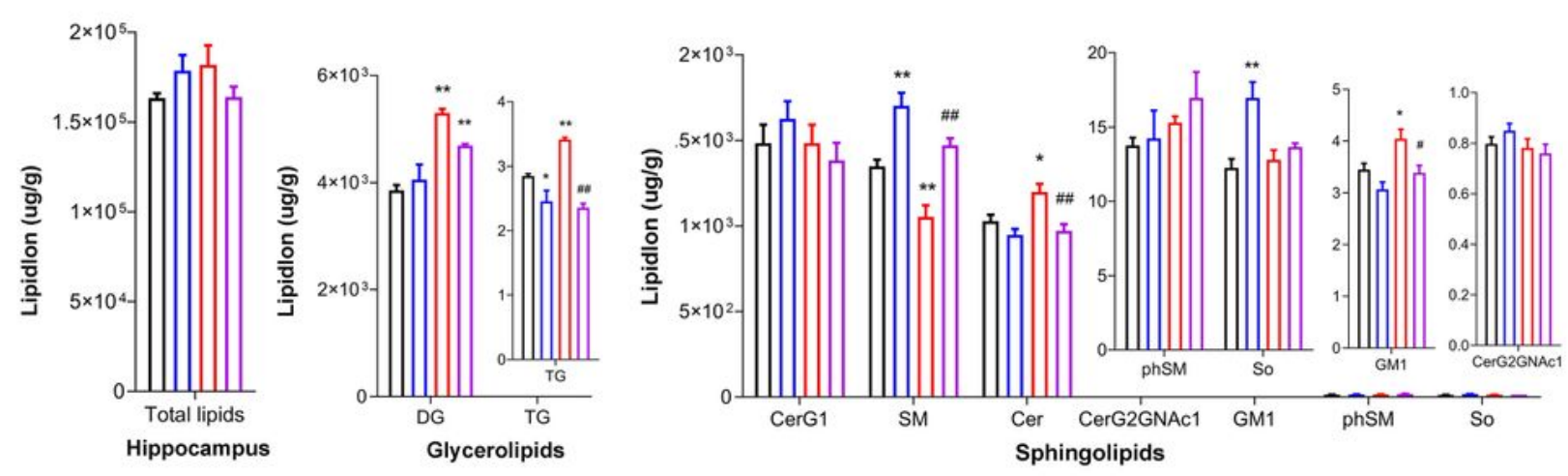

D

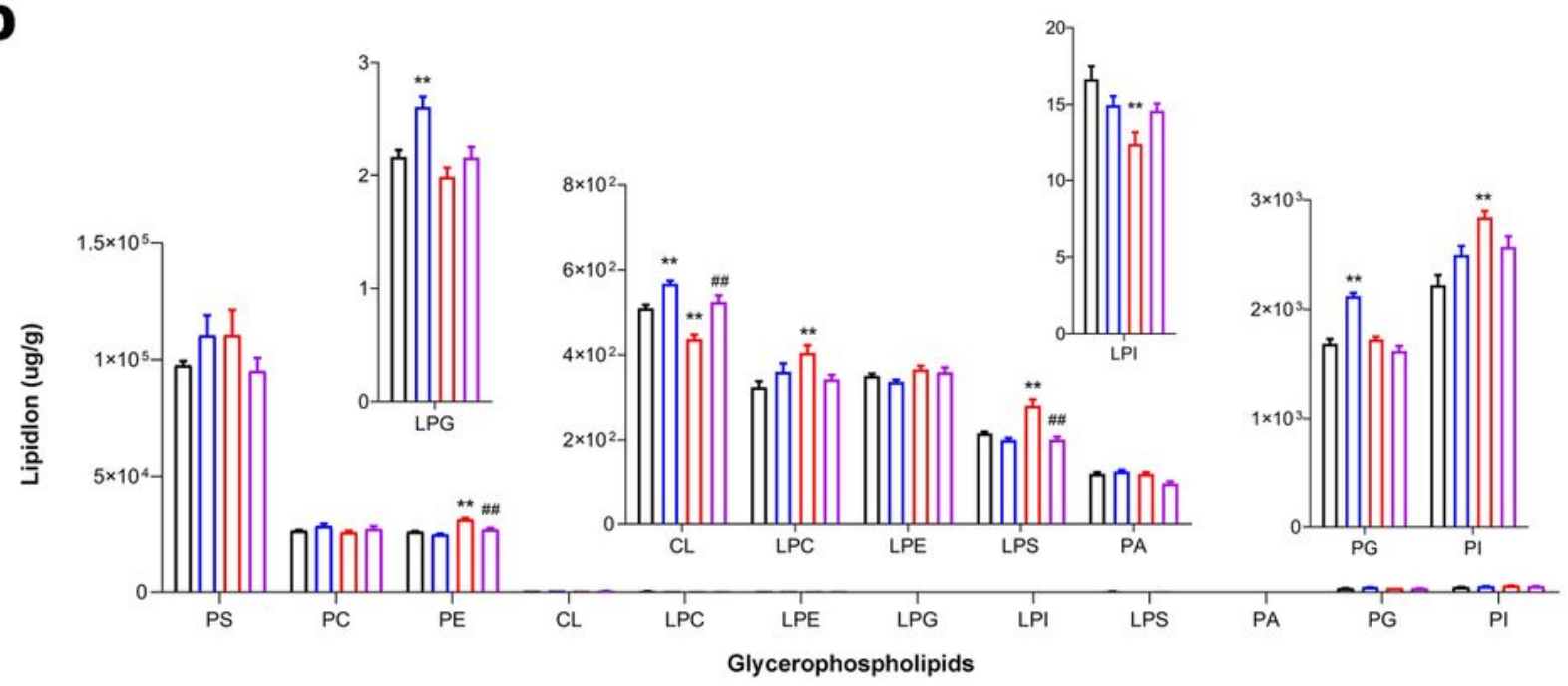

$\mathbf{E}$
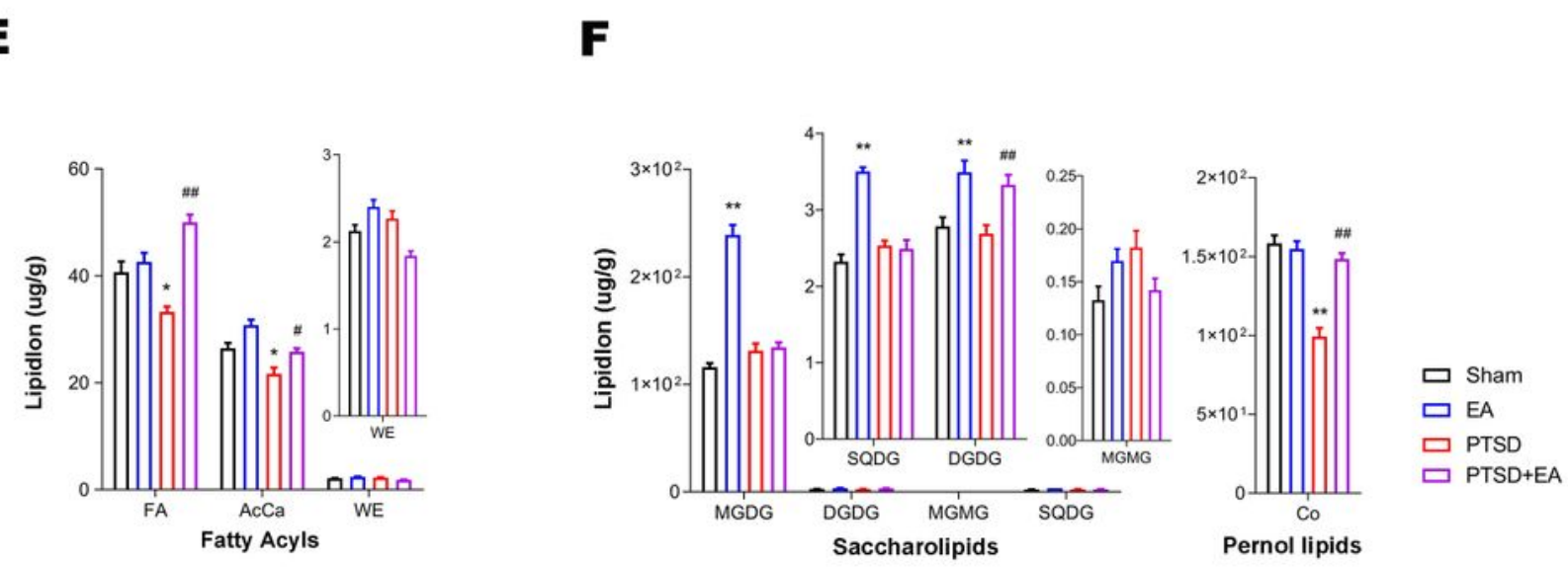

Figure 2

Groupwise alterations in the lipidomic profiles in the hippocampus. (A) Total lipid concentration, (B) glycerolipids, (C) sphingolipids, (D) glycerophospholipids, (E) fatty acyls, (F) saccharolipids and prenol lipids. DG, diglyceride; TG, triglyceride; CerG, glucosylceramides; SM, sphingomyelin; Cer, ceramides; GM1, 
gangliosides; phSM, phytosphingosine sphingomyelin; So, sphingosine; PS, phosphatidylserine; PC, phosphatidylcholine; $\mathrm{PE}$, phosphatidylethanolamine; $\mathrm{CL}$, cardiolipin; LPC, lysophosphatidylcholine; LPE, lysophosphatidylethanolamine; LPG, lysophosphatidylglycerol; LPI, lysophosphatidylinositol; LPS, lysophosphatidylserine; PA, phosphatidic acid; PG, phosphatidylglycerol; PI, phosphatidylinositol; FA, fatty acid; AcCa, acylcarnitine; WE, wax esters; MGDG, monogalactosyldiacylglycerol; DGDG, digalactosyldiacylglycerol; MGMG, monogalactosylmonoacylglycerol; SQDG, sulfoquinovosyldiacylglycerol; Co, coenzyme; PTSD, posttraumatic stress disorder; EA, electroacupuncture; ${ }^{*}, \mathrm{P}<0.05$ vs. Sham; **, $\mathrm{P}<0.01$ vs. Sham; \#, P< 0.05 vs. PTSD; \#\#, P< 0.01 vs. PTSD. 

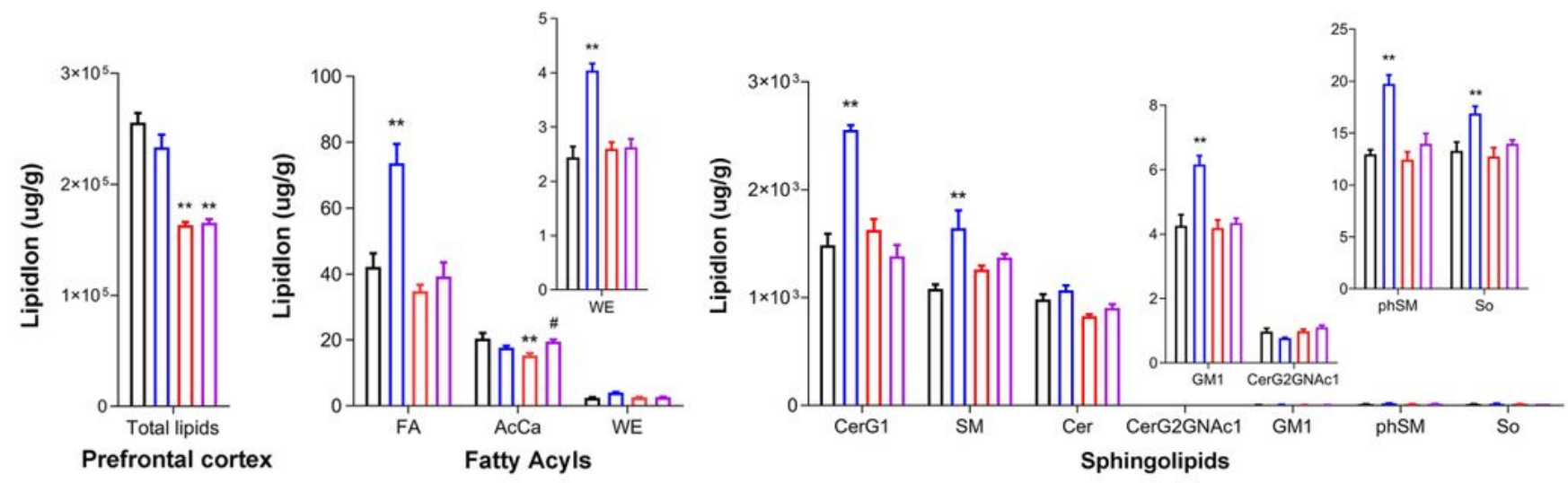

D

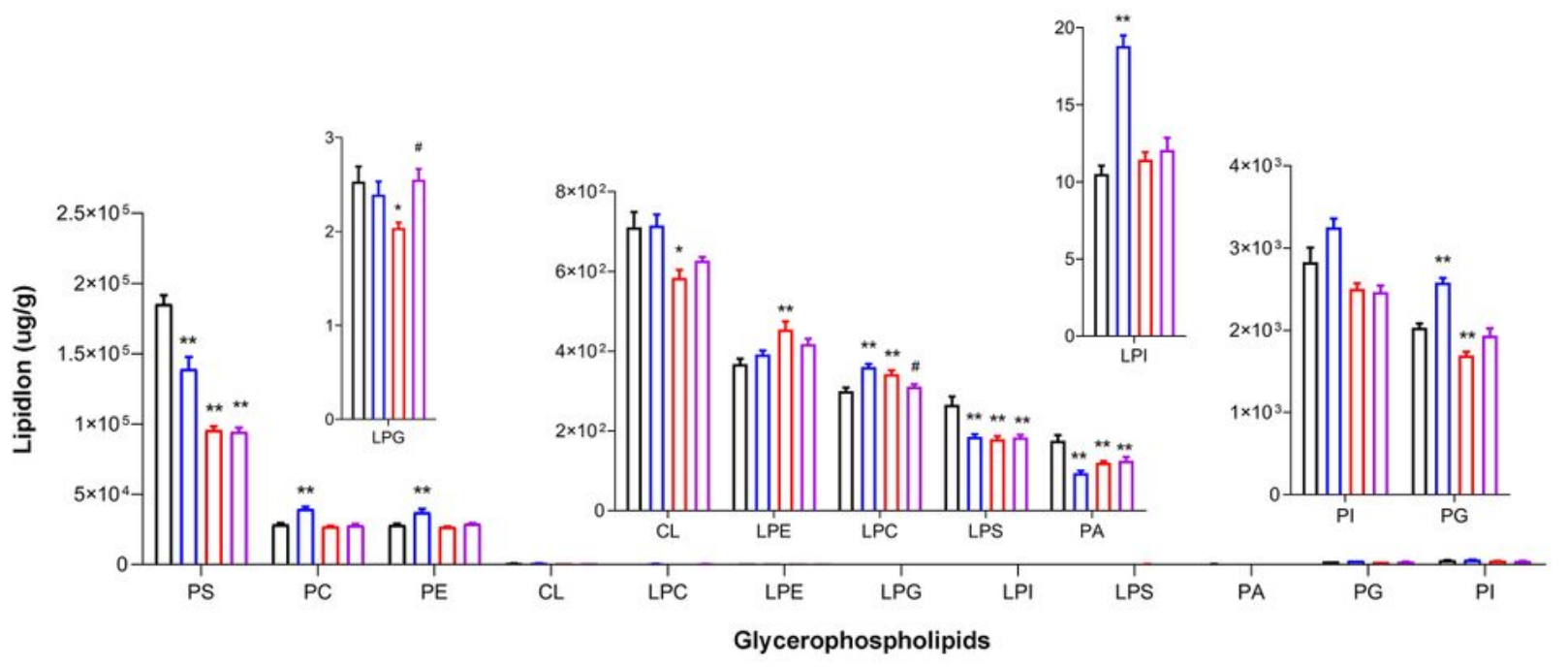

$\mathbf{E}$
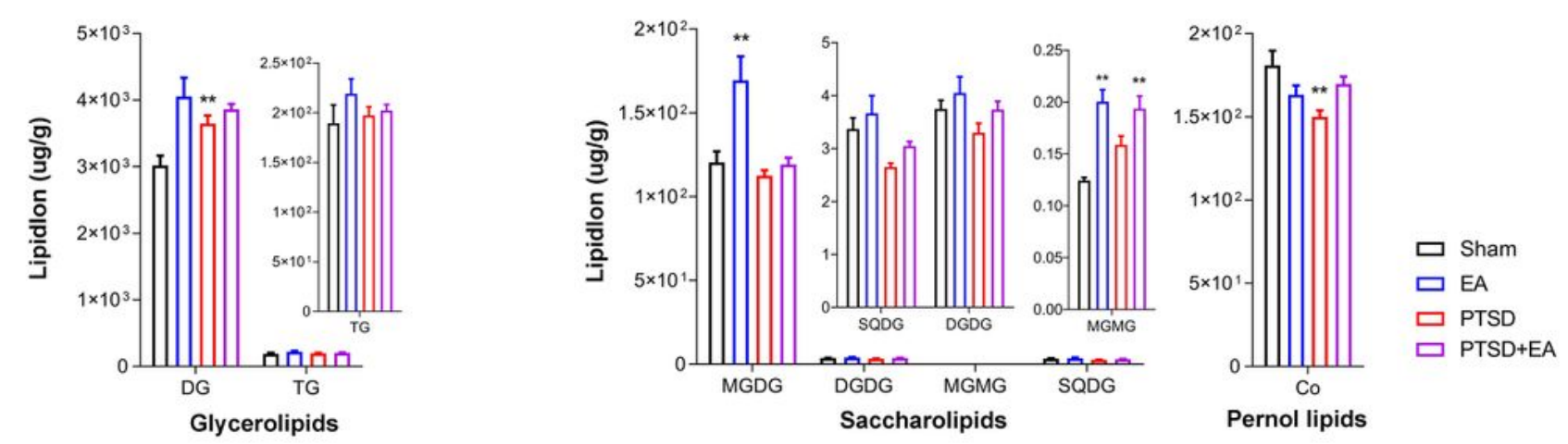

Figure 3

Groupwise alterations in the lipidomic profiles in the prefrontal cortex (PFC). (A) Total lipid concentration, (B) fatty acyls, (C) sphingolipids, (D) glycerophospholipids, (E) glycerolipids, (F) saccharolipids and prenol lipids. FA, fatty acid; AcCa, acylcarnitine; WE, wax esters; CerG, glucosylceramides; SM, sphingomyelin; Cer, ceramides; GM1, gangliosides; phSM, phytosphingosine sphingomyelin; So, sphingosine; PS, phosphatidylserine; PC, phosphatidylcholine; PE, phosphatidylethanolamine; $\mathrm{CL}$, 
cardiolipin; LPC, lysophosphatidylcholine; LPE, lysophosphatidylethanolamine; LPG, lysophosphatidylglycerol; LPI, lysophosphatidylinositol; LPS, lysophosphatidylserine; PA, phosphatidic acid; PG, phosphatidylglycerol; PI, phosphatidylinositol; DG, diglyceride; TG, triglyceride; MGDG, monogalactosyldiacylglycerol; DGDG, digalactosyldiacylglycerol; MGMG, monogalactosylmonoacylglycerol; SQDG, sulfoquinovosyldiacylglycerol; Co, coenzyme; PTSD, posttraumatic stress disorder; EA, electroacupuncture; *, $\mathrm{P}<0.05$ vs. Sham; ${ }^{\star \star}, \mathrm{P}<0.01$ vs. Sham; \#, $\mathrm{P}<$ 0.05 vs. PTSD.

A $r$ value

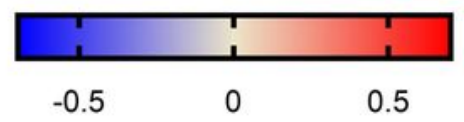

\section{Hippocampus}

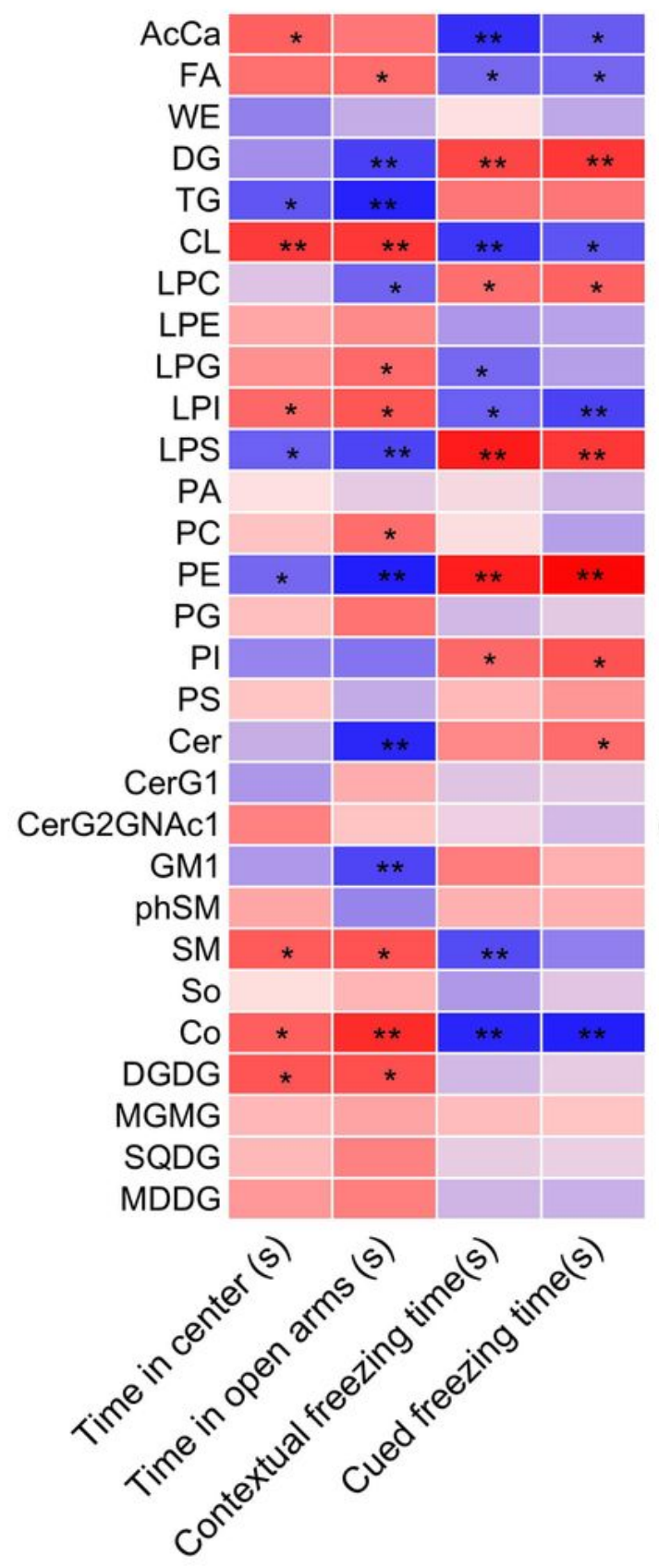

B

\section{Prefrontal cortex}

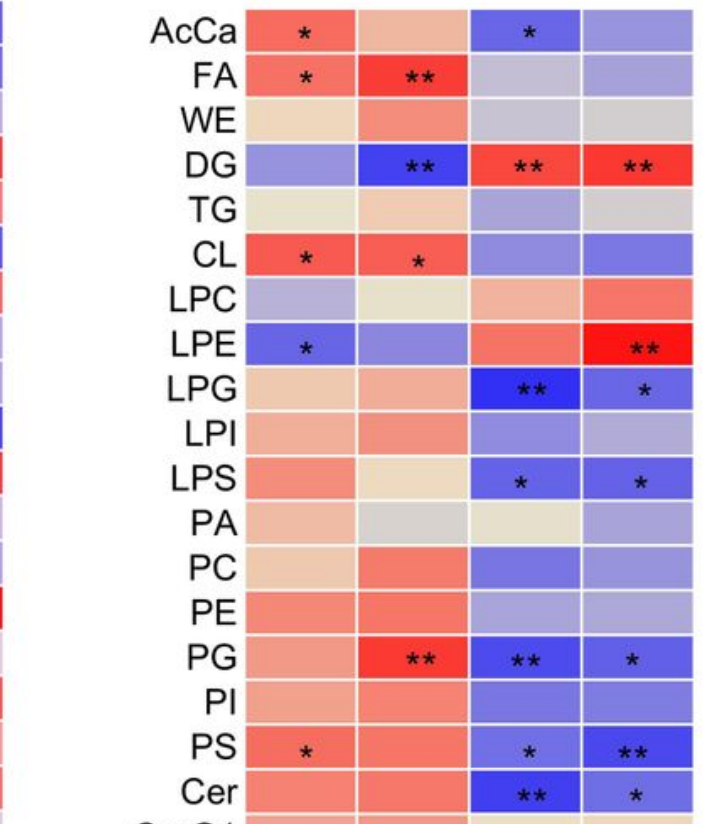

CerG2GNAC1

GM1

phSM

SM

So

Co

DGDG

MGMG

SQDG

MGDG

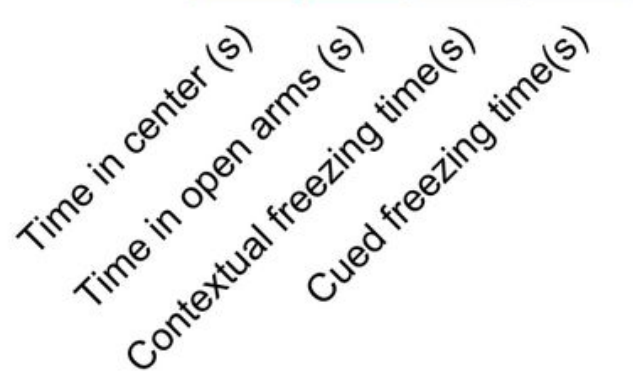


Figure 4

Analysis of correlations between PTSD-like behaviors and lipid levels in (A) the hippocampus and (B) the PFC, using Pearson's correlation coefficient. *, $\mathrm{P}<0.05 ; \star \star, \mathrm{P}<0.01$.

A

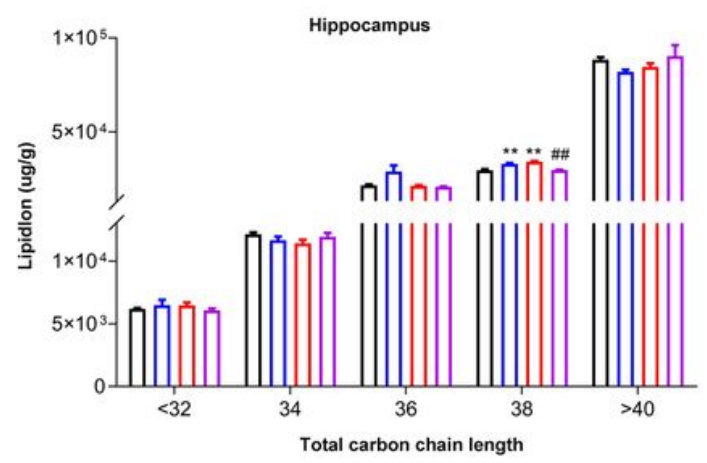

C

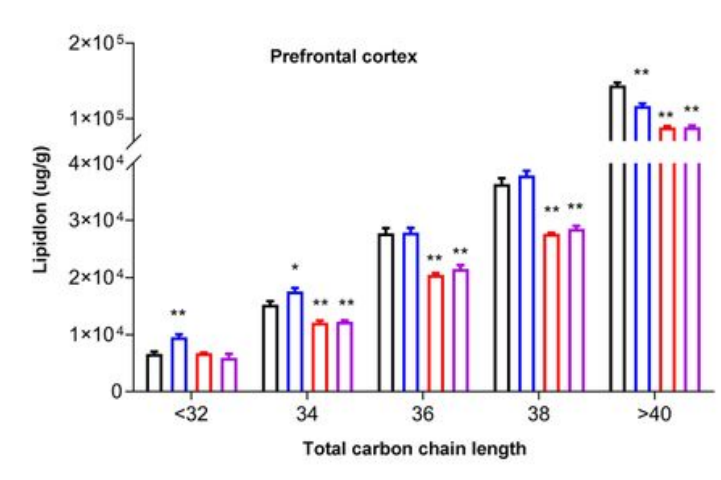

B

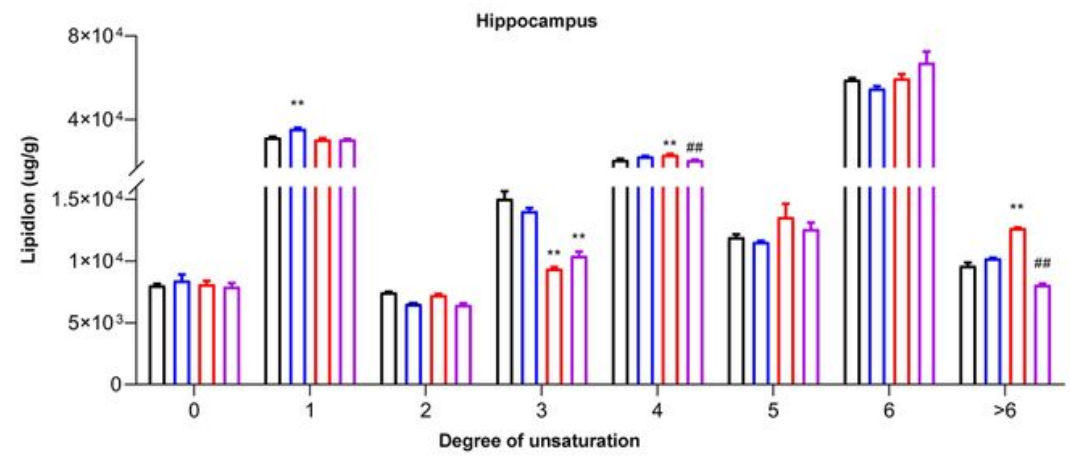

D

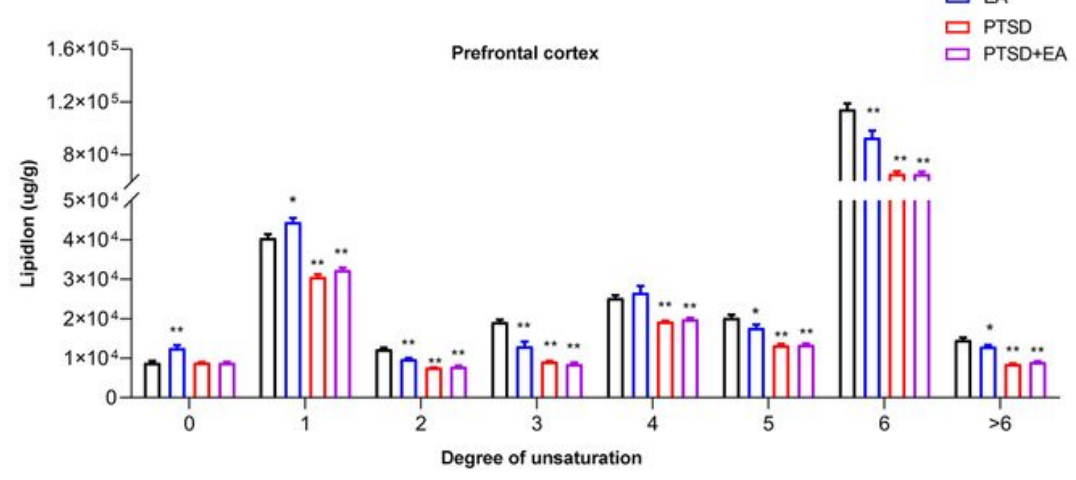

Figure 5

Groupwise alterations in fatty acid composition in the hippocampus and PFC. Results of analysis of fatty acyl composition by $(A, C)$ chain length (number of carbons) totals and $(B, D)$ degree of unsaturation totals. PTSD, posttraumatic stress disorder; EA, electroacupuncture; * $P<0.05$ vs. Sham; $* \star, P<0.01$ vs. Sham; \#, $P<0.05$ vs. PTSD; \#\#, $P<0.01$ vs. PTSD. 
A

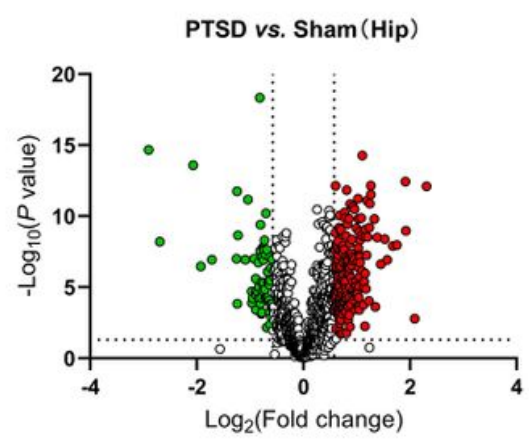

C

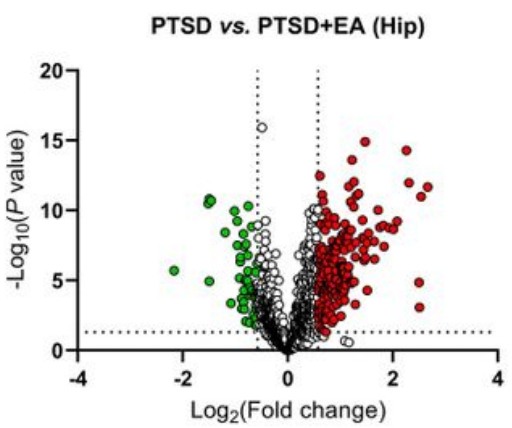

E

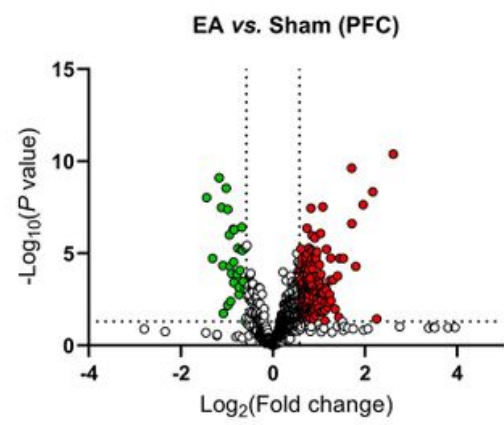

B

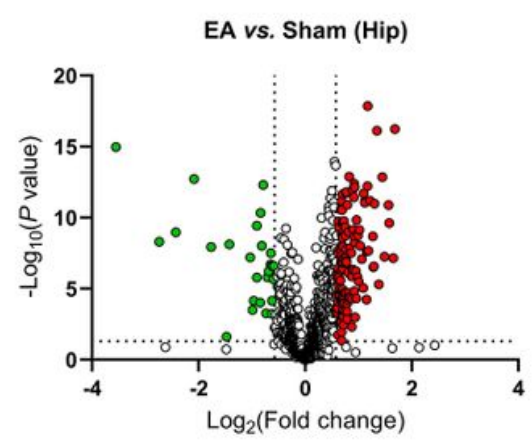

D

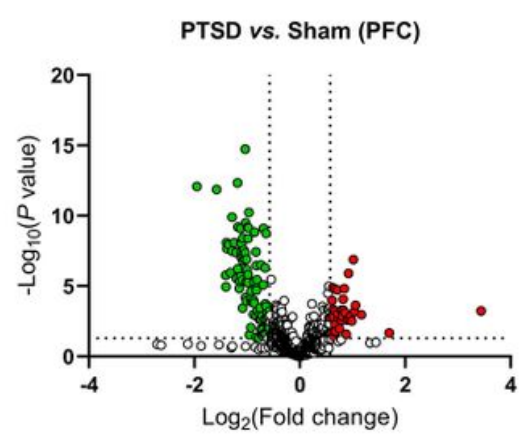

F

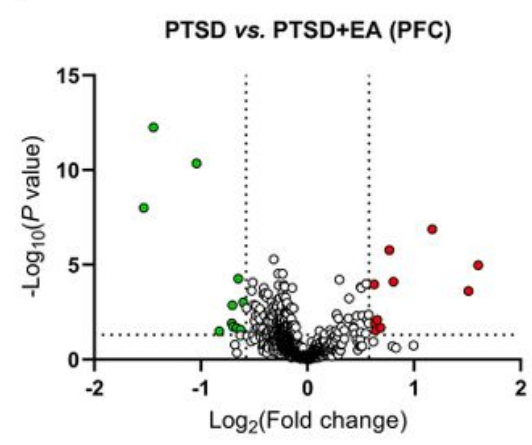

G

$r$ value
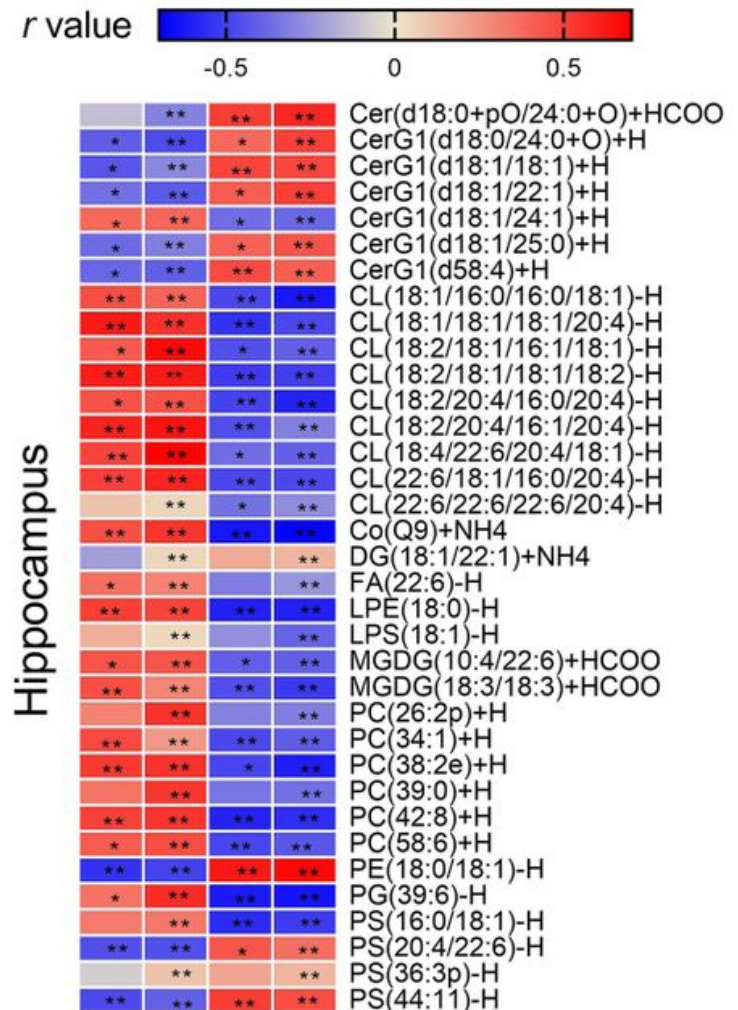


\section{Supplementary Files}

This is a list of supplementary files associated with this preprint. Click to download.

- sumpplementaltable1.docx

- sumpplementaltable2.docx

- sumpplementaltable3.docx

- sumpplementaltable4.docx

- sumpplementaltable5.docx

- sumpplementaltable6.docx 